\title{
Addressing Minority Stress and Mental Health among Men Who Have Sex with Men (MSM) in China
}

\author{
Shufang Sun ${ }^{1} \cdot$ John E. Pachankis ${ }^{2} \cdot{\text { Xiaoming } \mathrm{Li}^{3} \cdot \text { Don Operario }}^{4}$ \\ Published online: 16 January 2020 \\ (C) Springer Science+Business Media, LLC, part of Springer Nature 2020
}

\begin{abstract}
Purpose of Review Men who have sex with men (MSM) in China experience elevated risks of mental health issues in comparison to the general population in China, which contribute to vulnerability to HIV/STI risks and can comprise the effectiveness of HIV prevention efforts. A conceptual framework for understanding this mental health disparity is minority stress theory, which posits that experiences of external prejudice events (i.e., distal stressors) and internal stress processes such as internalized homophobia and concealment (i.e., proximal stressors) contribute to sexual minorities' elevated risk of psychological distress. To deepen the understanding of mental health among Chinese MSM and explore the potential utility of minority stress theory in this population, this paper synthesizes research evidence regarding prevalent mental health issues as well as how minority stress may be linked to psychological health in Chinese MSM.

Recent Findings Results indicate that Chinese MSM experience a high prevalence of several mental health issues including depression, anxiety, suicidal behaviors, and alcohol dependence.

Summary This review further reveals minority stress to be an important determinant of psychological distress among Chinese MSM, though evidence is mixed regarding the relationship between proximal minority stress and psychological health. Nonetheless, there is a lack of mental health services and interventions focusing on MSM in China. Culturally relevant, competent, and LGBT-affirmative mental health interventions are needed for Chinese MSM. To guide future intervention research, we provide considerations for reducing minority stress and promoting psychological health among Chinese MSM.
\end{abstract}

Keywords China $\cdot$ Men who have sex with men $\cdot$ Minority stress $\cdot$ Stigma $\cdot$ Psychological health

\section{Introduction}

Globally, men who have sex with men (MSM) are disproportionately burdened by poor mental health [1-3]. Across

This article is part of the Topical Collection on The Global Epidemic

Shufang Sun

shufang_sun@brown.edu

1 Department of Psychiatry and Human Behavior, Brown University Alpert Medical School, 167 Point St, Providence, RI 02906, USA

2 Department of Social and Behavioral Sciences, Yale School of Public Health, New Haven, Connecticut, USA

3 Department of Health Promotion, Education, and Behavior, University of South Carolina Arnold School of Public Health, Columbia, SC 29208, USA

4 Department of Behavioral and Social Sciences, Brown School of Public Health, Providence, RI, USA geographic regions, studies consistently find that MSM experience elevated rates of mood and anxiety disorders as well as a higher burden of alcohol and substance use disorders compared to their heterosexual peers [4-9]. Such mental health disparities contribute to "syndemic" conditions surrounding HIV infection risk behaviors $[10,11]$ and interfere with the uptake of HIV-related behavioral interventions among MSM [12]. Mental health issues are particularly concerning among MSM in China, the world's most populous country. Along with the significant uptrend in the HIV epidemic among MSM in China [13, 14], recent reports suggest that Chinese MSM also suffer from a variety of mental health issues, including depression, anxiety, suicidal ideation, and alcohol dependence [15-18].

Similar to their western counterparts, Chinese MSM largely live in a heteronormative, stigmatizing environment [19, 20]. One explanation for the mental health disparities in sexual minorities is minority stress theory, which posits that stressors induced by a homophobic environment specific to sexual 
minority status can lead to adverse psychological outcomes for sexual minorities $[21,22]$. Clear and consistent evidence supports this theory in MSM, suggesting that minority stressrelated experiences such as discrimination, victimization, and internalized homophobia contribute to psychological distress and stress-sensitive illness [23-28]. However, this evidence is primarily established in Western, predominately US-based samples, which limits the potential utility of this theory to understand and address the burden of mental health in MSM in the global context.

Despite the growing HIV epidemic in Chinese MSM, recent reports on mental health concerns, and increased research interests in this population, empirical evidence on mental health and the role of minority stress among Chinese MSM has not been synthesized. This review paper, thereby motivated by the need to address this gap in the literature, aims to articulate a comprehensive understanding of minority stress and mental health among Chinese MSM and offer recommendations for future mental and behavioral health interventions targeting Chinese MSM. By gaining a minority stressinformed understanding of mental health in Chinese MSM, this research synthesis may also offer insight into the adaptation and application of this perspective in efforts to improve to the mental health of MSM in other global regions. Thus, the key scientific inquiry leading this review is how minority stress may be linked to Chinese MSM's psychological health. To answer this question, we structure the review of research findings in three sections: (1) overview on empirical evidence on mental health issues among Chinese MSM; (2) minority stress as a determinant of Chinese MSM's mental health; and (3) mental health services and relevant interventions for Chinese MSM. After synthesizing research findings in the above areas, we provide recommendations on future interventions aiming at reducing minority stress and improving mental health among Chinese MSM.

\section{Search Strategy}

A comprehensive literature search was conducted using PyscINFO, ProQuest, SAGE, and Google Scholar. The search was limited to papers published in the past 6 years (from 2013 to 2019). Similar to previous reviews on Chinese MSM [29], articles published in peer-reviewed English language journals are emphasized so that readers can access them. We used search terms including minority stress, stigma, discrimination, mental health, depression, anxiety, China, men who have sex with men, men who have sex with men and women, gay, bisexual men, and sexual minority men. Quantitative research findings are emphasized, with qualitative studies included as supplemental materials to provide relevant context and supportive evidence. To be included, studies needed to focus on MSM in China or report findings from a subsample of MSM in China. Studies also needed to measure and report mental health in Chinese MSM. In summary, the following inclusion criteria were employed: (1) focus on Chinese MSM; (2) published or in press from 2013 to May 2019; (3) written in English; and (4) measured and reported mental health.

Table 1 presents the details and key findings of the 37 studies focused on mental health among MSM in China identified through this search. Some studies $(n=26,70.3 \%)$ targeted HIV-negative, serostatus-unknown, or serostatusvaried MSM whereas others $(n=11,29.7 \%)$ focused on MSM living with or newly diagnosed with HIV. The majority of the studies used nonprobability sampling, including snowball sampling ( $n=15,40.5 \%)$ and convenience sampling $(n=$ $20,54.1 \%$ ), usually in the context of HIV and MSM-related community outreach in HIV prevention and care. Stratified random sampling was used in two studies (5.4\%). Seven studies' recruitment methods also included online-based efforts as part of their recruitment methods $(18.9 \%)$. The majority of studies were cross-sectional ( $n=36,97.3 \%)$, and one study $(2.7 \%)$ was longitudinal. Most studies investigated mental health of MSM using a within-group approach by focusing on $\operatorname{MSM}(n=35 ; 94.6 \%)$, while two studies $(5.4 \%)$ compared MSM to their heterosexual peers in a larger sample.

\section{Empirical Evidence Regarding Mental Health Issues among Chinese MSM}

In this section, we review extant evidence documenting prevalent mental health concerns to shed light on potential mental health disparities that MSM in China experience and offer targets for interventions. Below, we summarize study findings concerning prevalent mental health issues in Chinese MSM. We also review study findings documenting the heterogeneity of Chinese MSM and subpopulations with particularly high rates of mental health problems, including MSM living with HIV.

Measurement of Mental Health In terms of mental health measurement, most studies $(n=35 ; 94.6 \%)$ employed nonface-to-face, survey methods, using scales such as the Symptoms Checklist-90 (SCL-90) and the Center for Epidemiological Studies-Depression (CES-D) scale. In-person, diagnostic interviews were employed in two study publications (derived from the same dataset), using the Composite International Diagnostic Interview (CIDI). This nonpopulation-based study with 807 MSM in Northeast China found a $35.2 \%$ lifetime prevalence of psychiatric disorders [17], which is more than twice as high compared to $16.6 \%$ in the general Chinese population [77]. In this sample, among MSM who met criteria for at least one diagnosis, $29.2 \%$ and $37.6 \%$ had comorbid disorders during the past 12 months and 


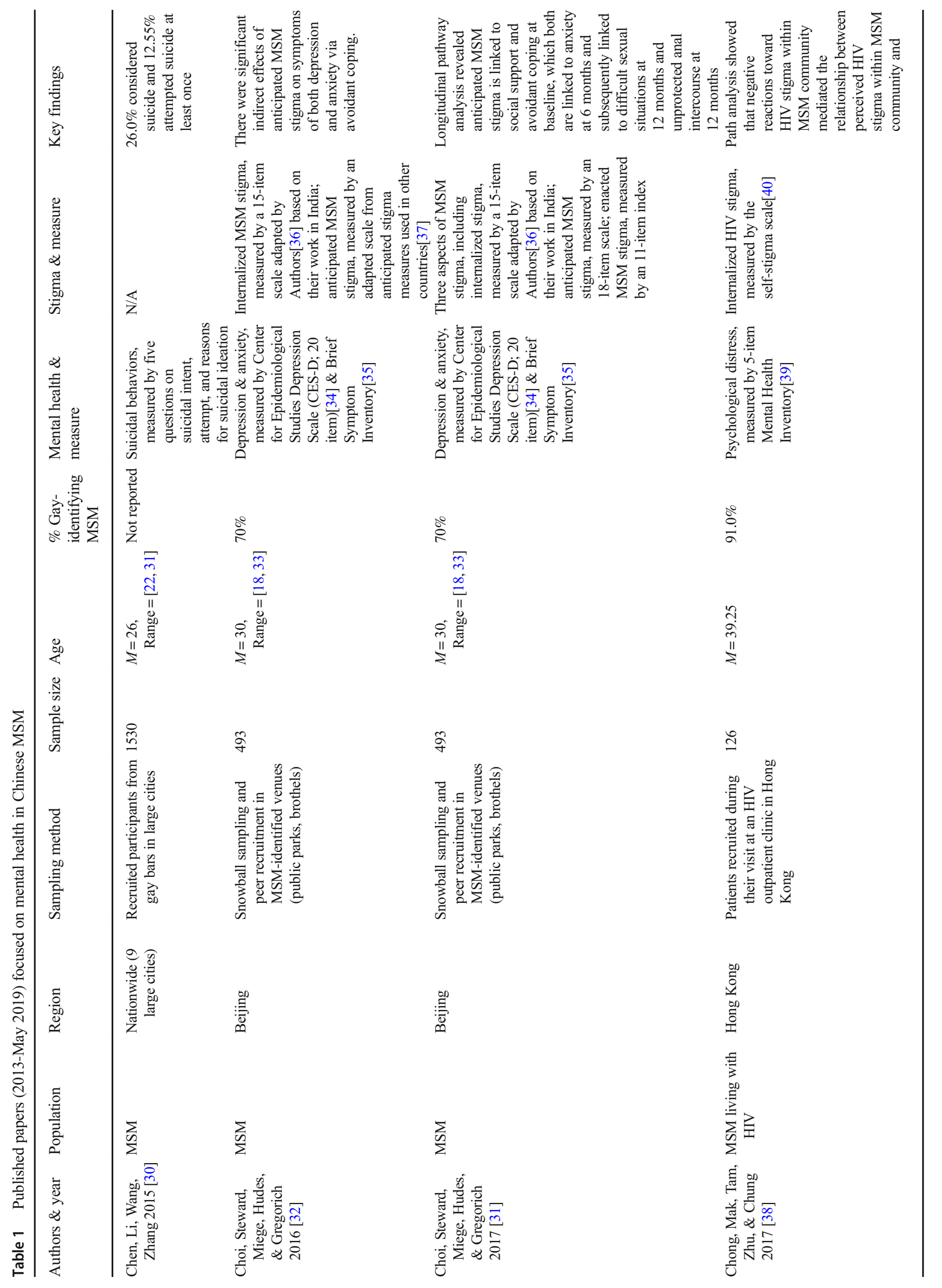




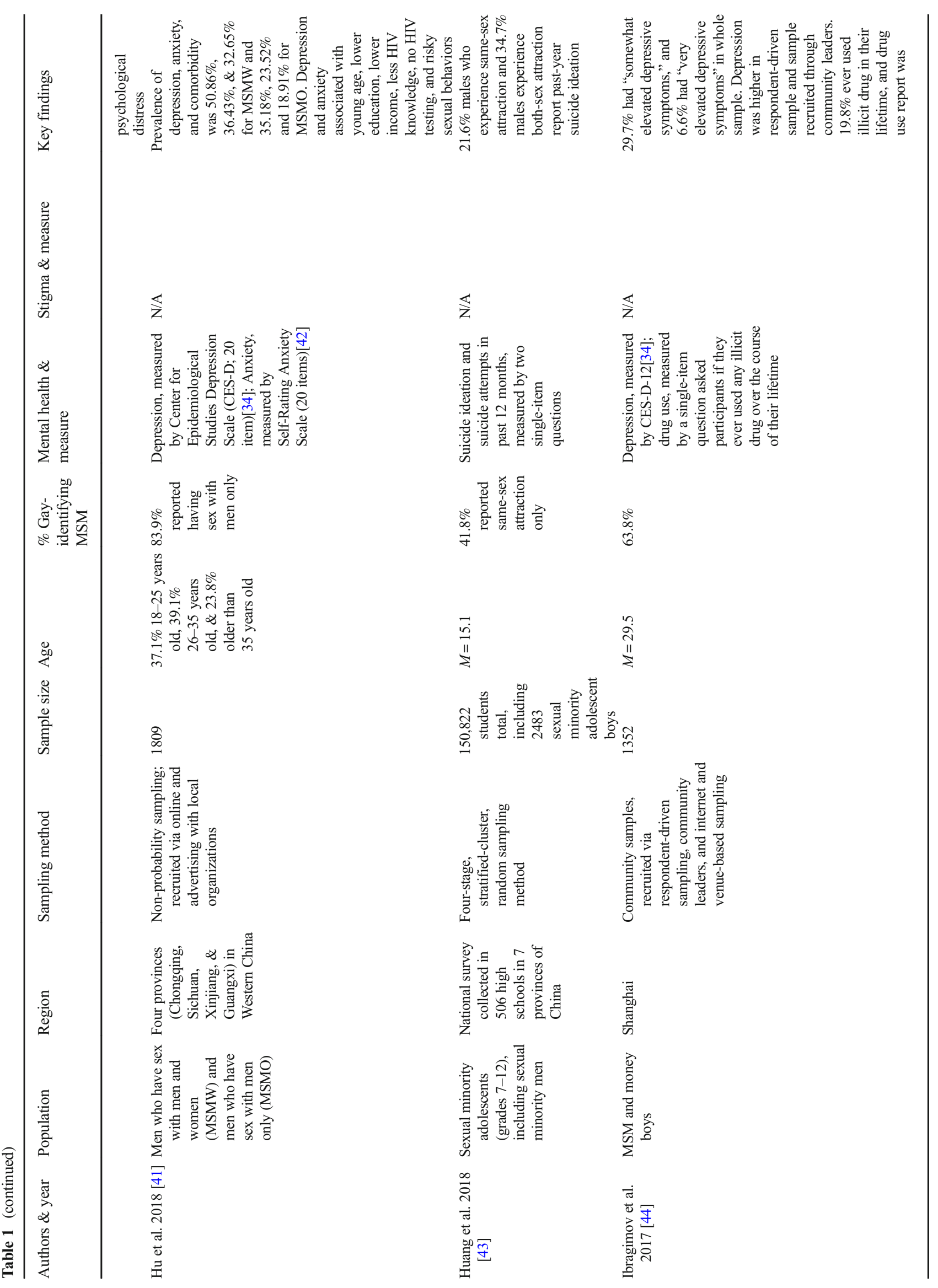




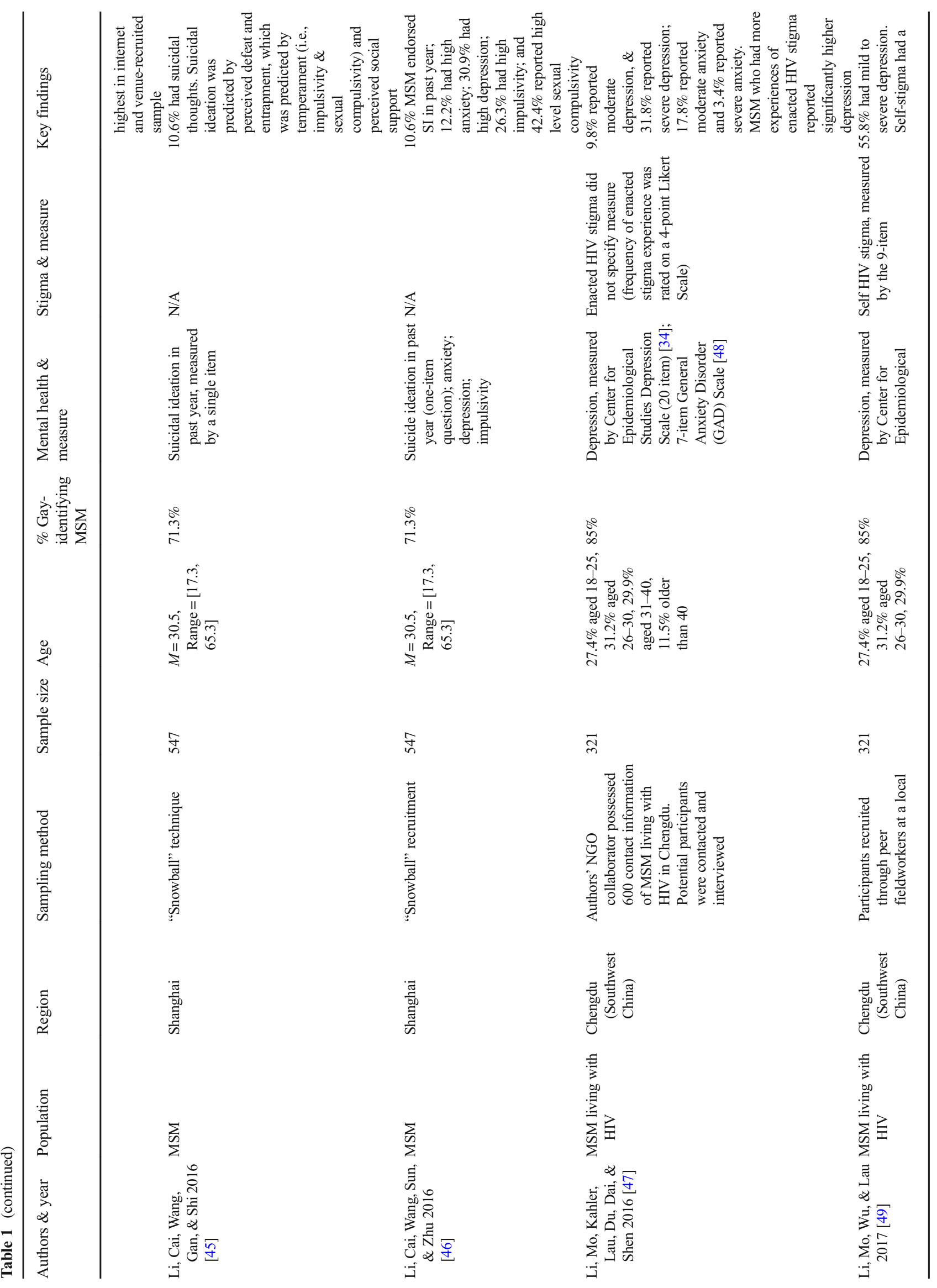




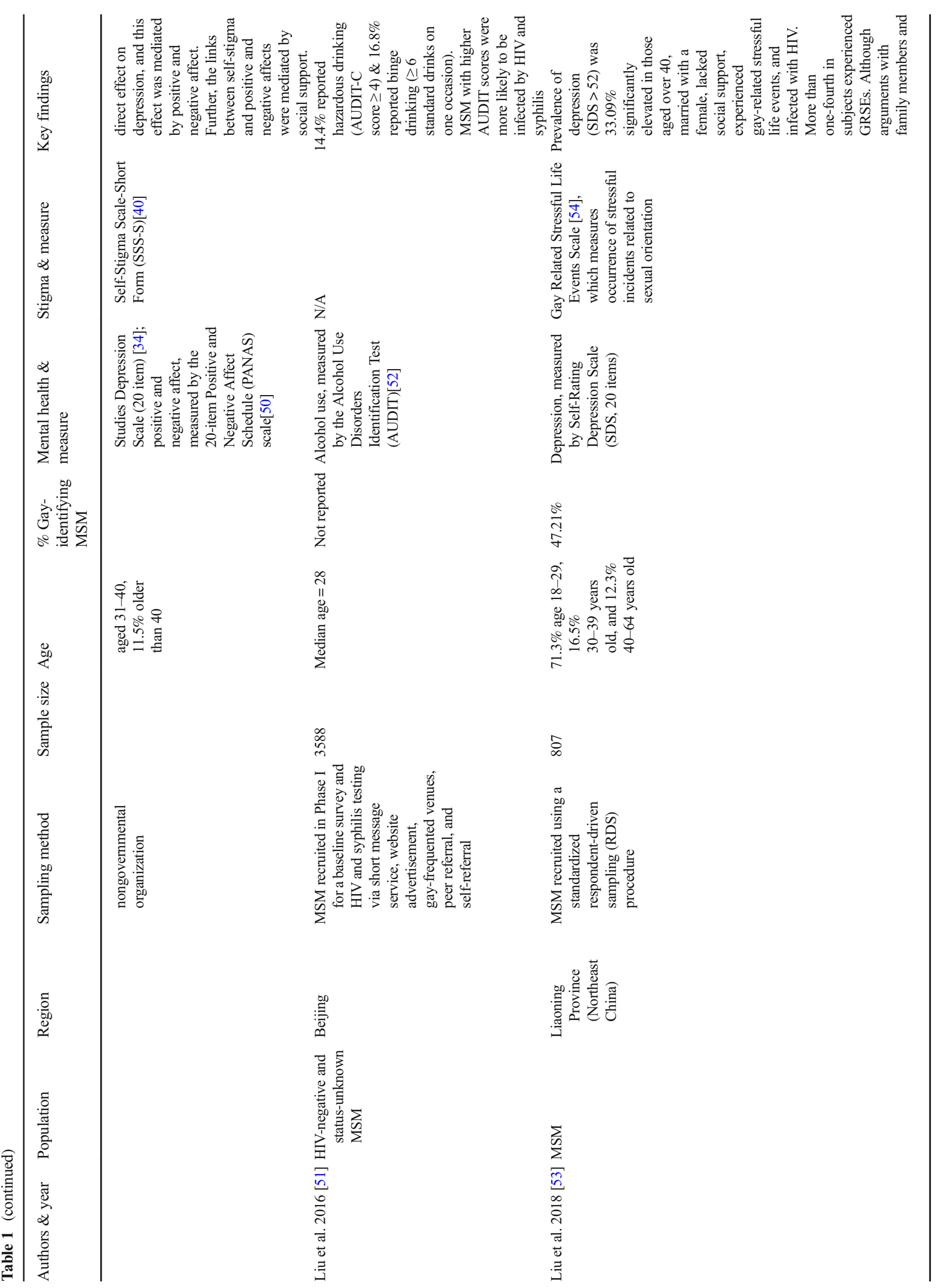




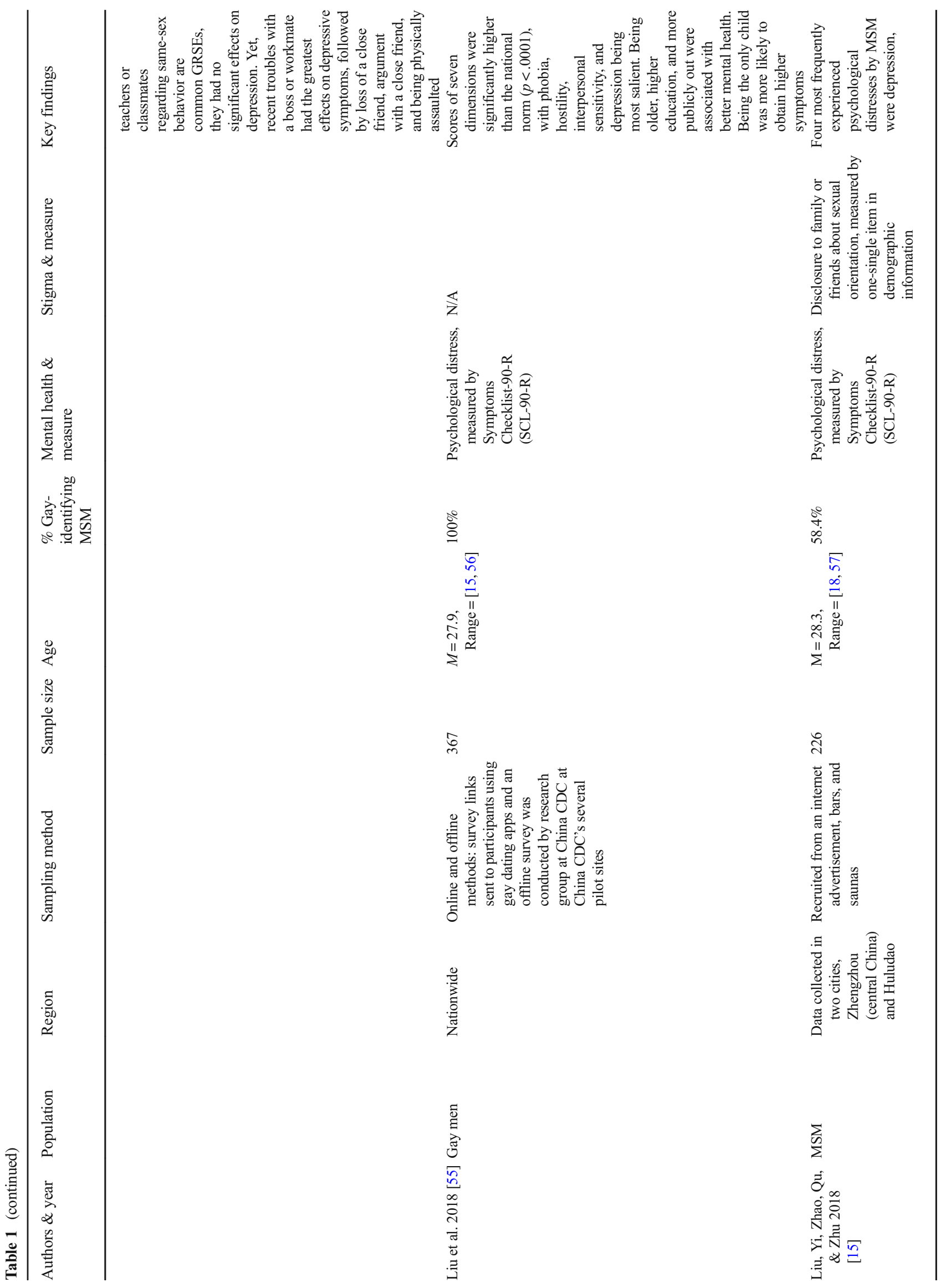




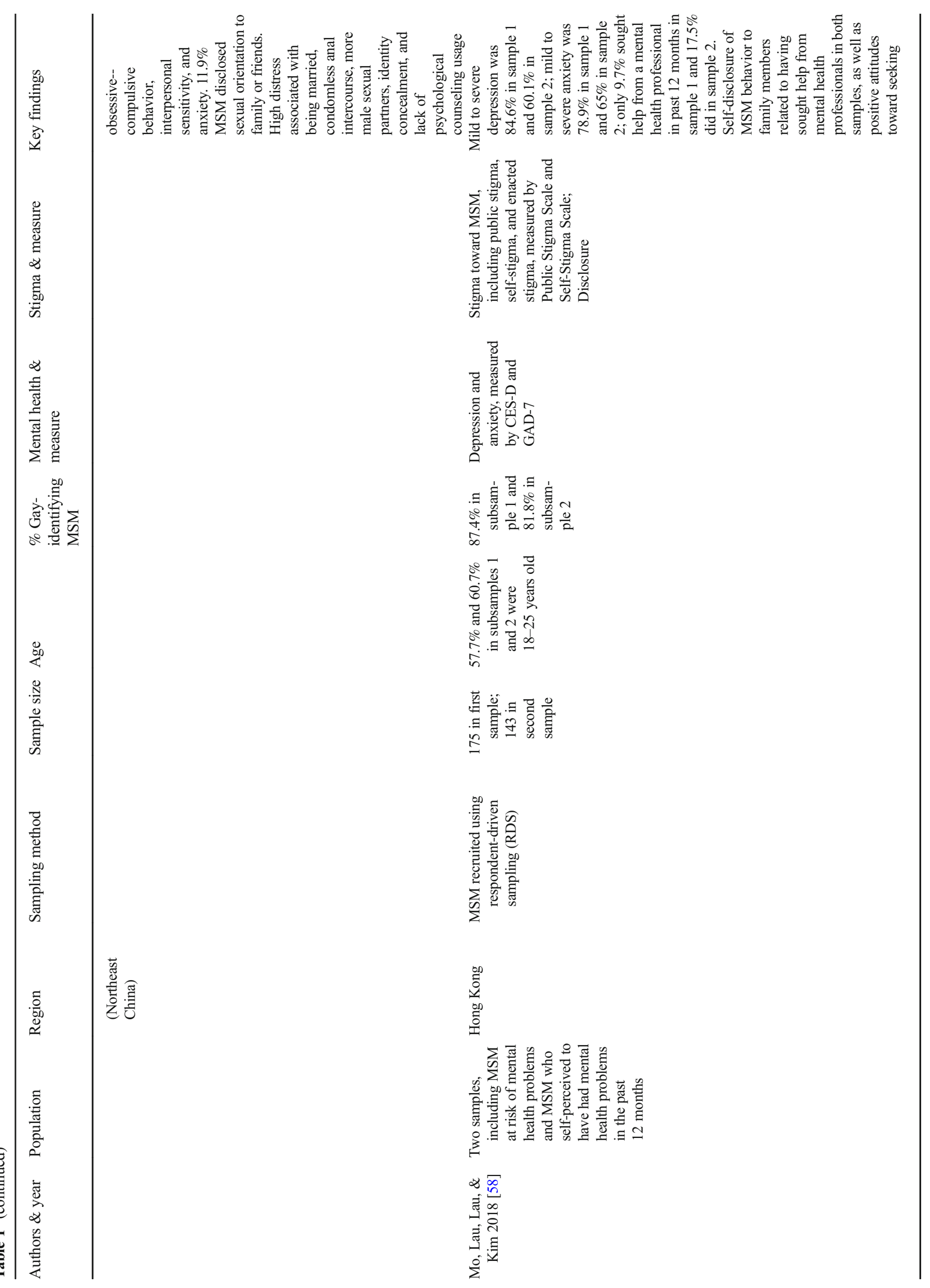




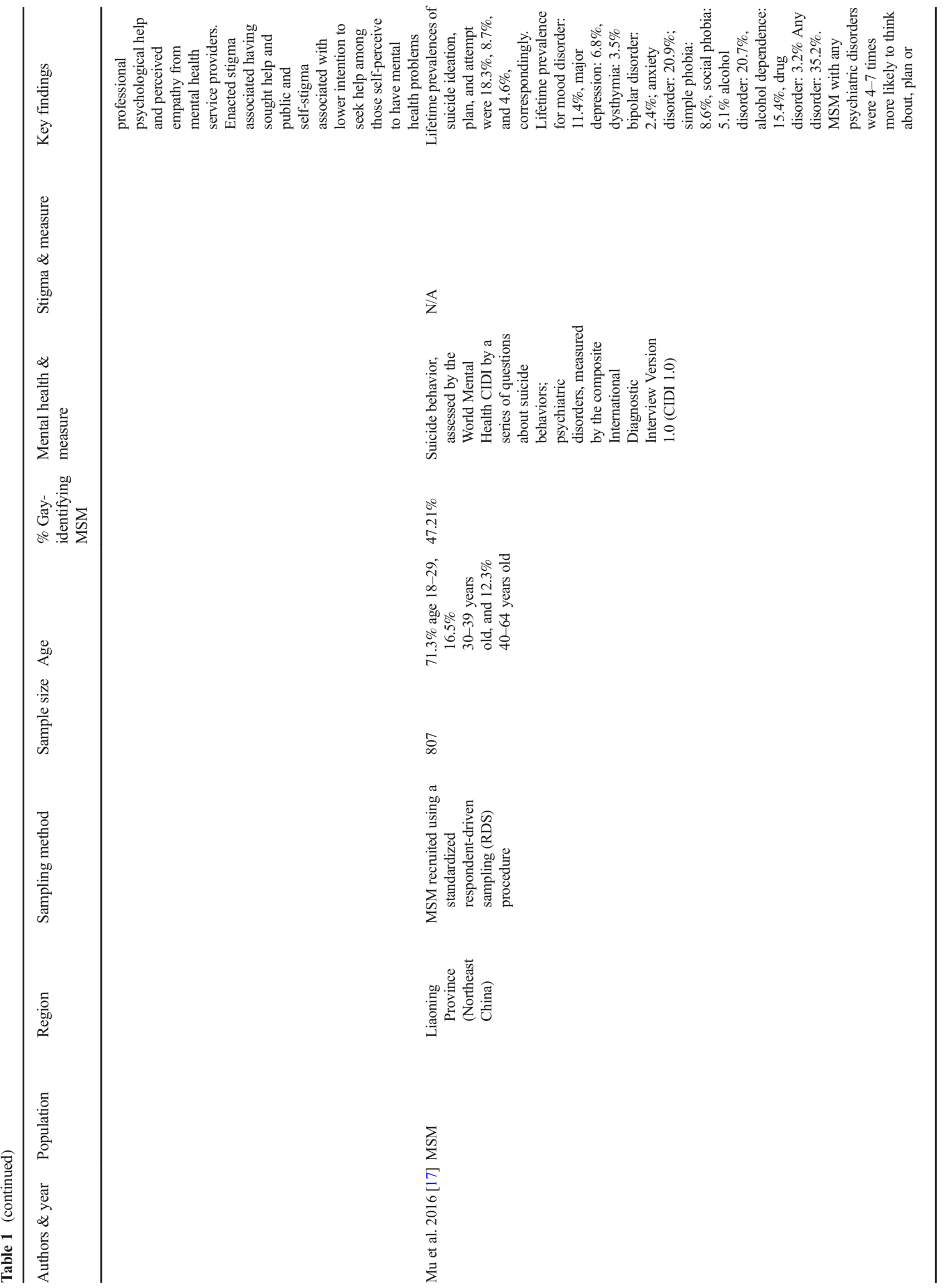




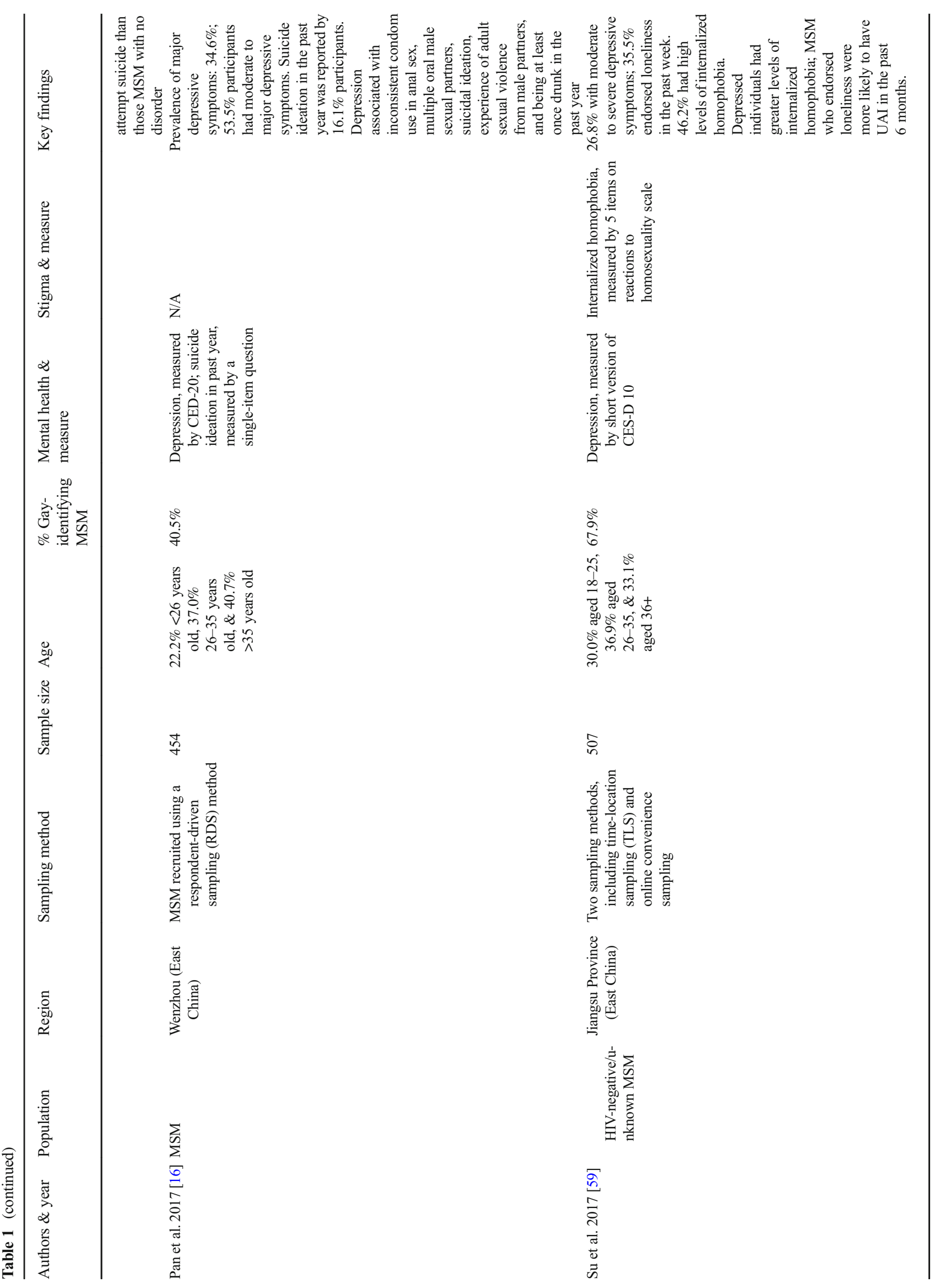




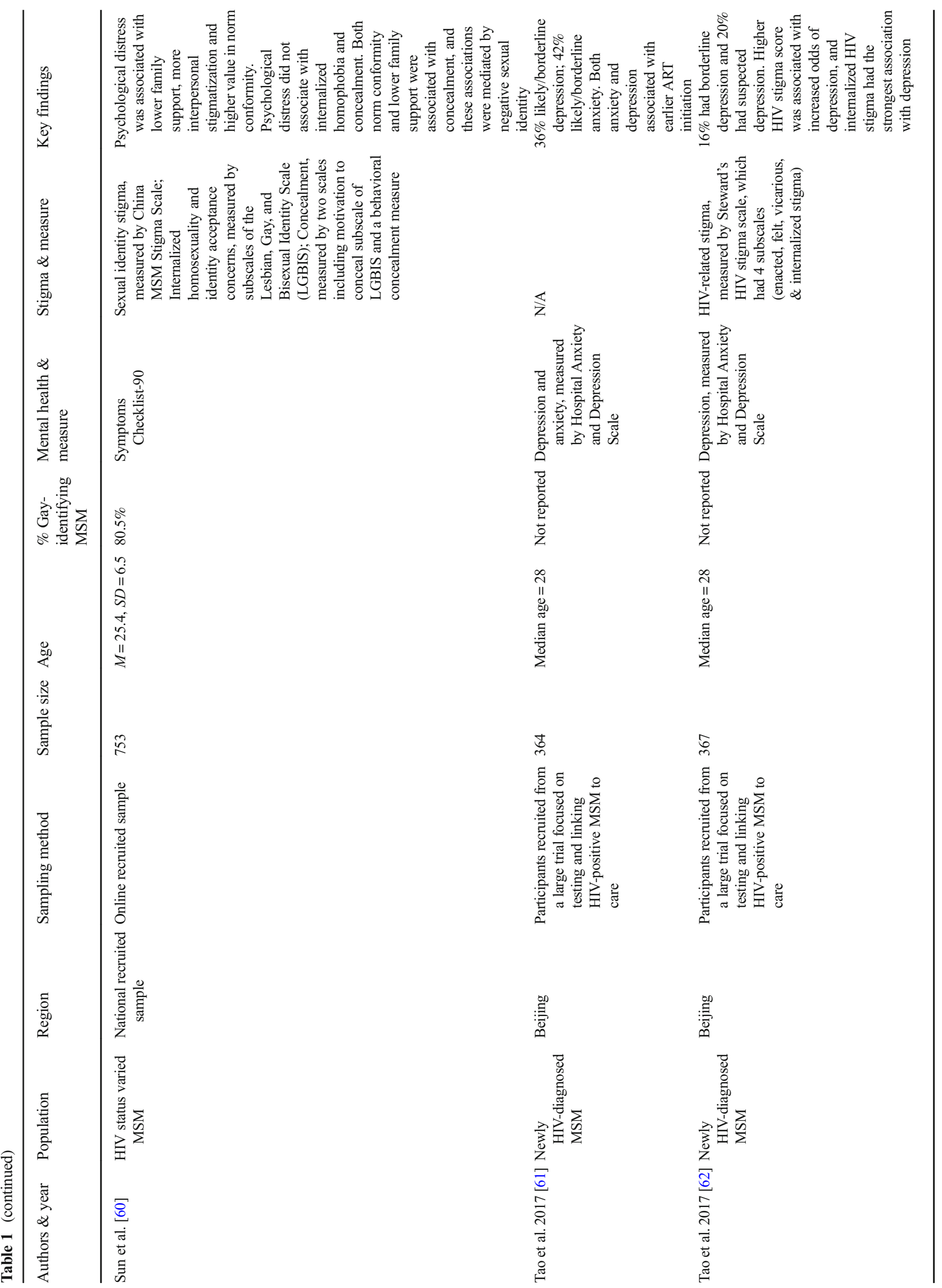




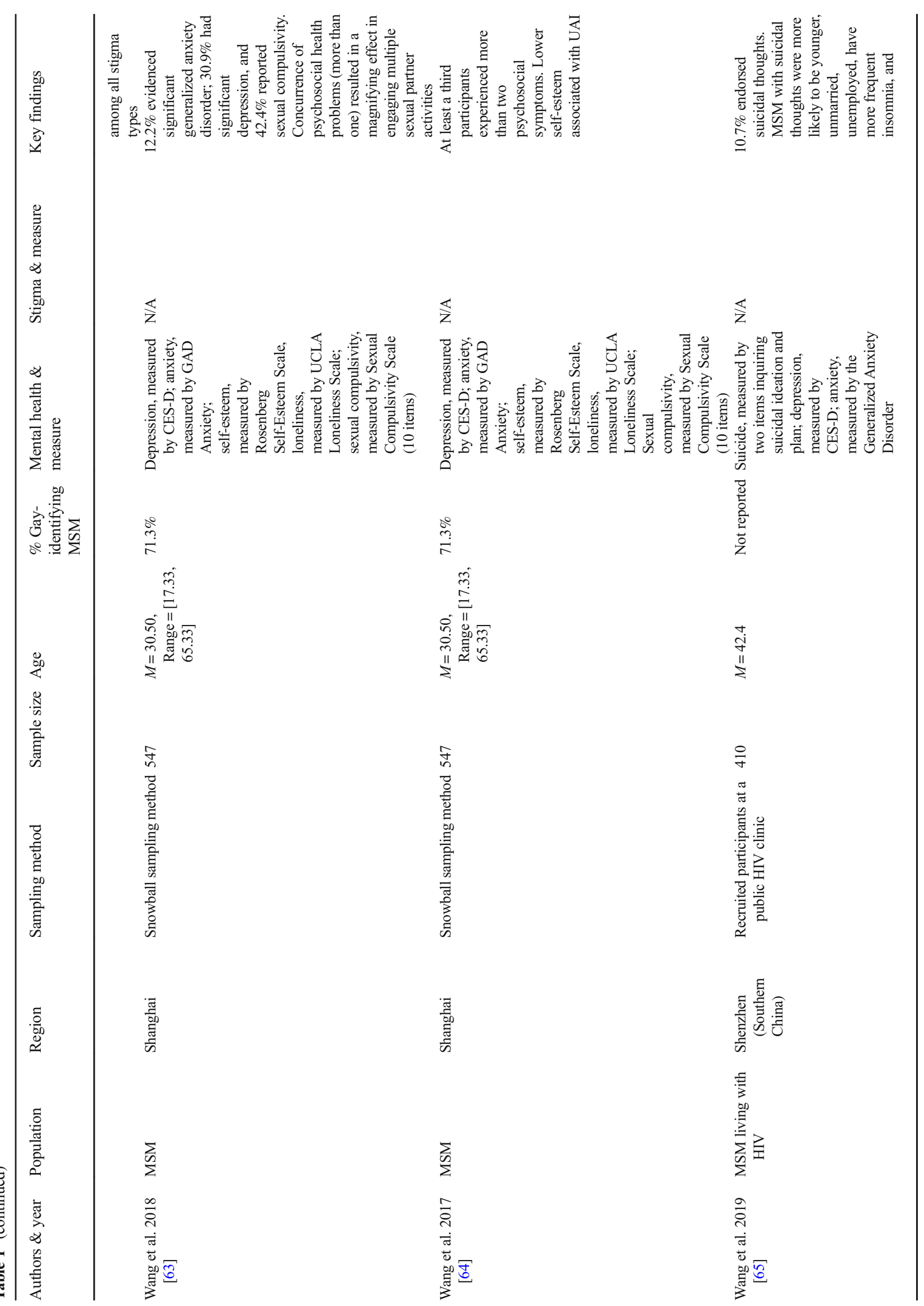




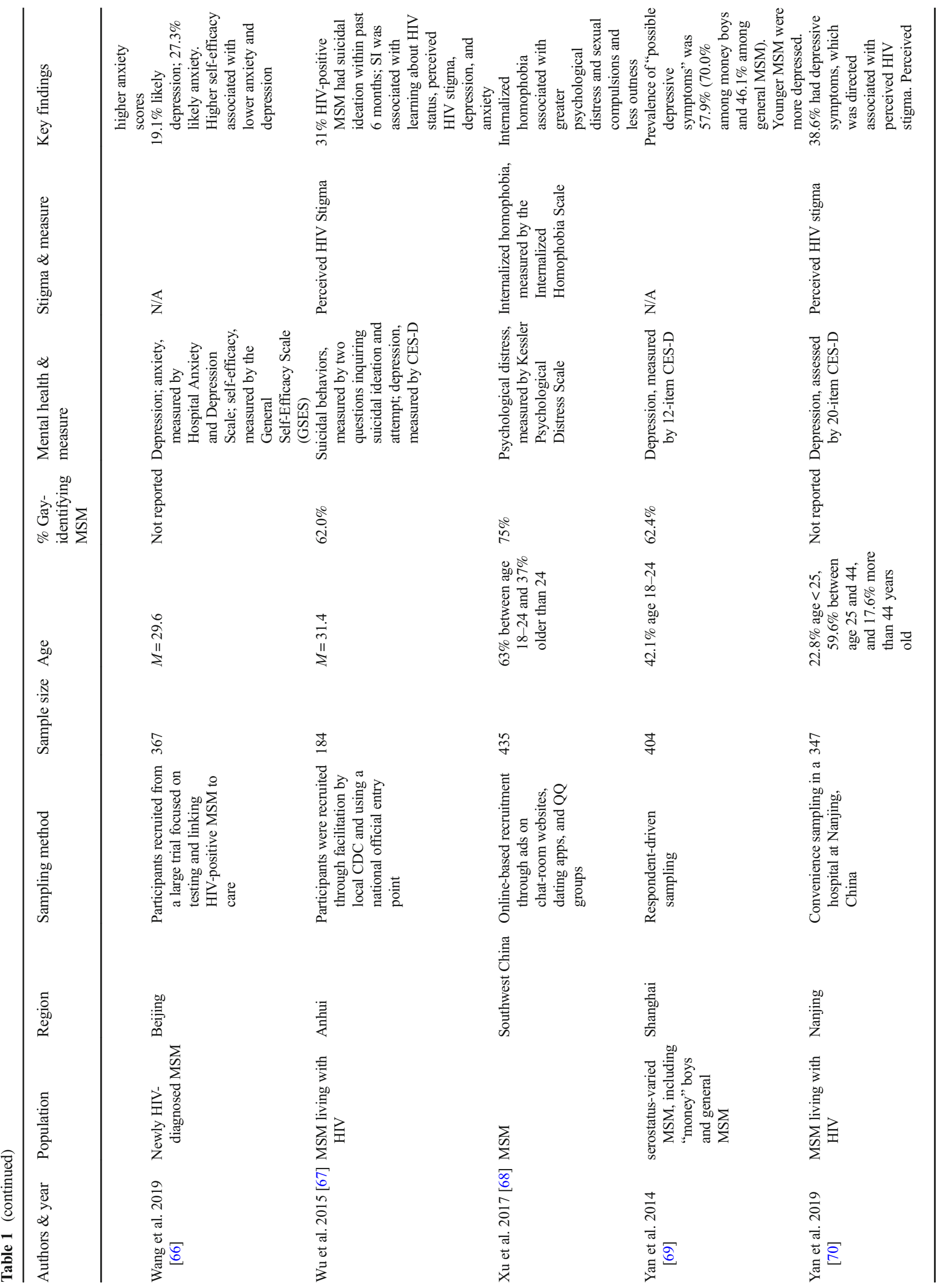




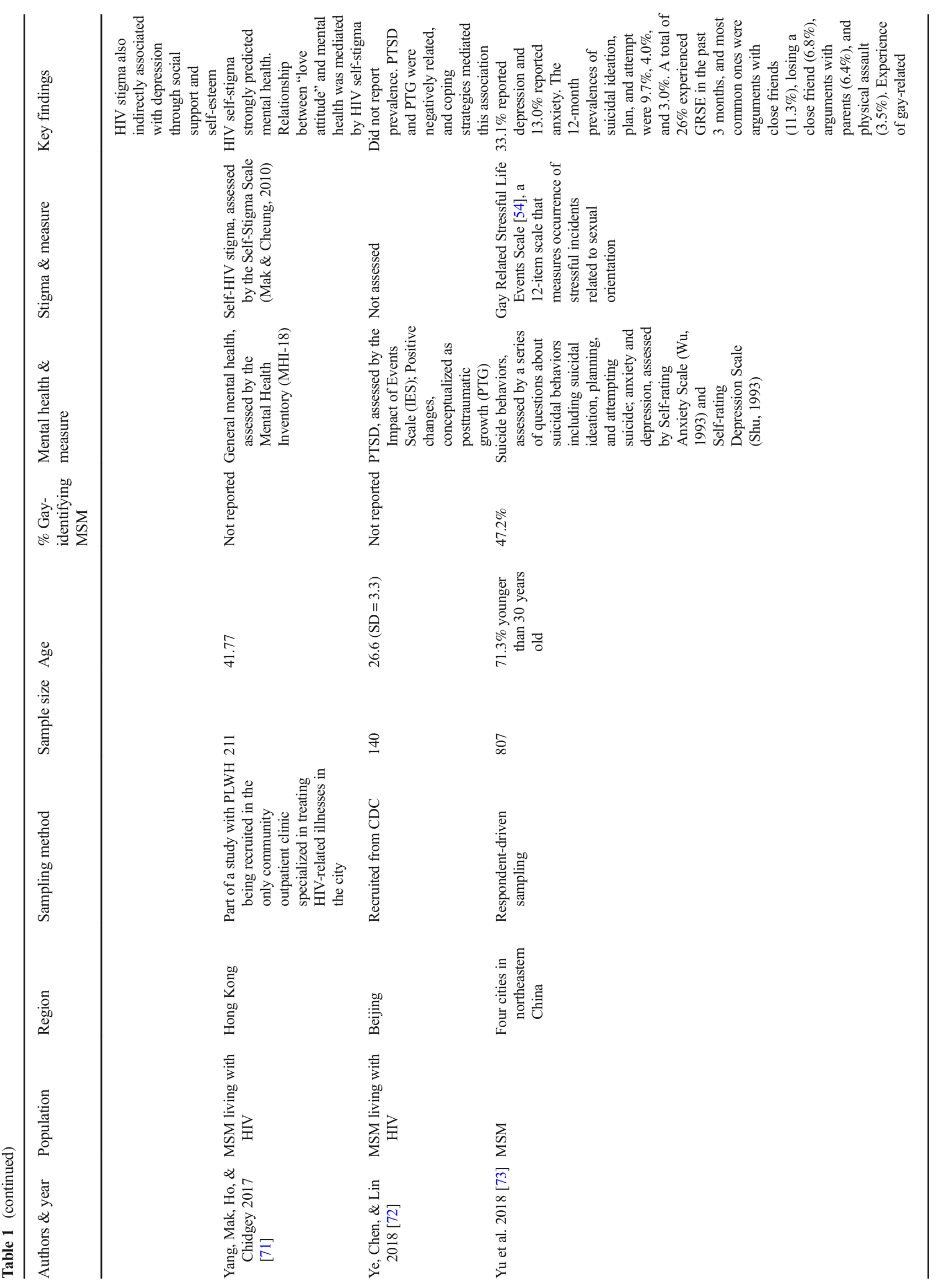




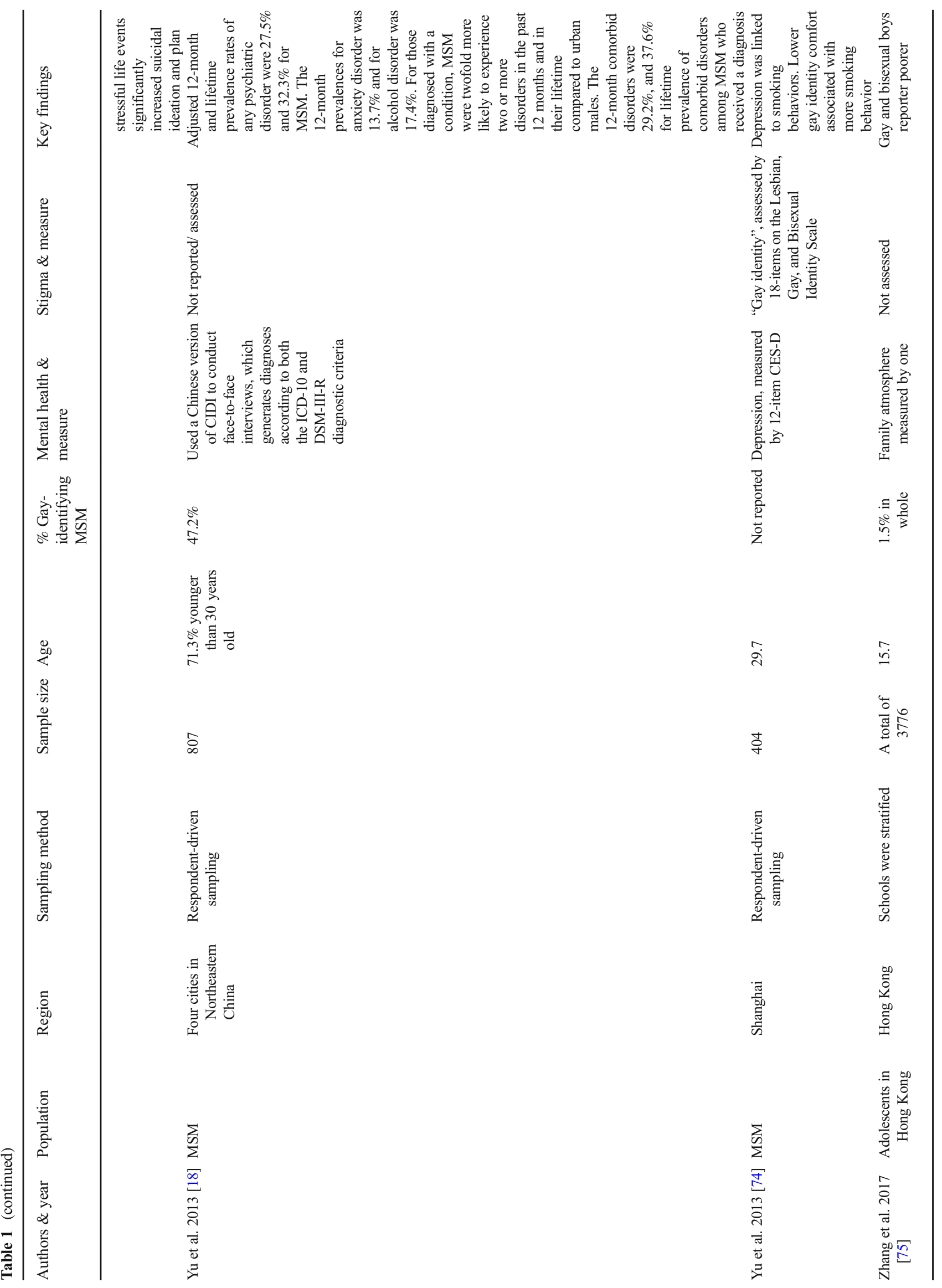




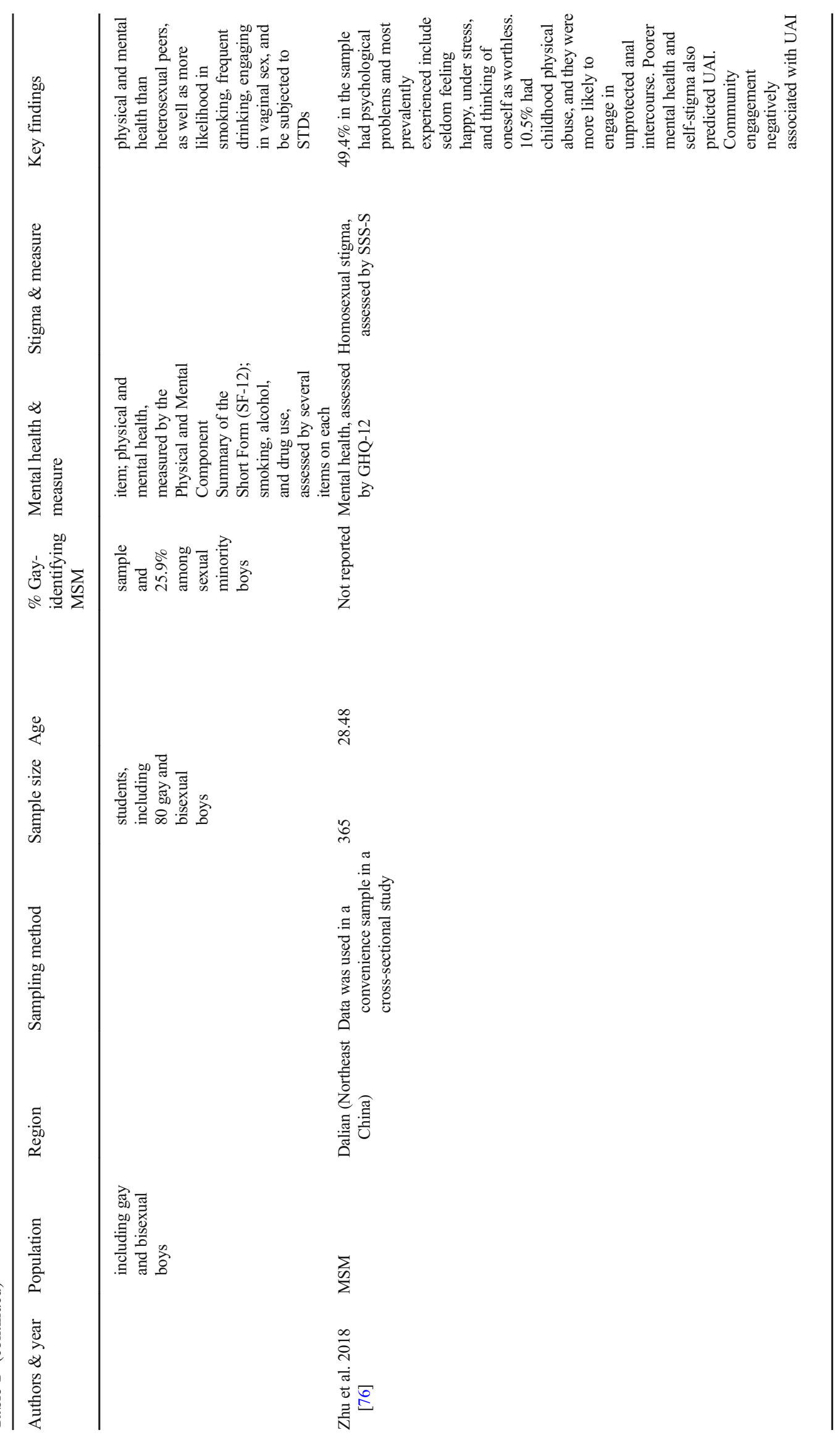


lifetime, respectively, equivalent to double the comorbidity risk compared to their urban male counterparts in China [18].

Notably, none of the identified studies assessed trauma symptoms (e.g., post-traumatic stress disorder symptoms), which therefore is not documented in this review. Psychological trauma may be a potentially fruitful area for future investigation, given that literature in the USA and other western societies has documented that MSM experience elevated risk for PTSD symptoms, which is associated with minority stress and linked to HIV-related risk behaviors [78-80].

Depression Depression is among the most researched mental health issues among Chinese MSM. Among studies of Chinese MSM of varied HIV status, moderate-to-severe depressive symptoms have been documented with varied rates ranging from $26.8 \%$ [59] to $53.5 \%$ [16]. Depression increases the vulnerability of HIV for Chinese MSM, such that those who suffer from depressive symptoms are more likely to engage in inconsistent condom use during anal sex [16, 31, 59, 64].

Anxiety Survey studies that report anxiety symptoms among samples of serostatus-varied Chinese MSM found anxiety symptoms to be prevalent (varying from $12.2 \%$ [46] to $25.6 \%$ [41]) but with higher rates in exclusive samples of MSM living with HIV (21.2\% [47] to 42\% [61]). A study that employed diagnostic interviews documented anxiety disorders as the most prevalent lifetime mental disorder among Chinese MSM (20.9\%; [17]). In a longitudinal study with MSM in China, anxiety predicted subsequent difficult sexual situations (i.e., settings and situations that made it difficult for one to practice safe sex such as sex within interpersonal relationships of unequal power) and condomless anal intercourse [31].

Suicidal Behaviors Studies have repeatedly documented that sexual minorities in western countries are more vulnerable to suicidal behaviors than their heterosexual peers. A recent meta-analysis summarizing results in the USA, Canada, Europe, Australia, and New Zealand concluded that $11 \%$ of sexual minority adults have attempted suicide in their lifetime compared to $4 \%$ of heterosexual adults in population surveys [81]. Similarly, suicidal behaviors are significantly elevated among Chinese MSM compared to the general population. Studies in this review reported varying prevalence of lifetime suicide attempt in Chinese MSM varied from 4.6\% [17] to $12.6 \%$ [30], compared to an estimated $0.8 \%$ prevalence of suicide attempt in the general Chinese population in a reported recent meta-analysis documenting studies from 2007 to 2013 [82]. For HIV status-varied Chinese MSM, the prevalence of suicidal ideation has been documented to be between $10.6 \%$ [45] and as high as $26.0 \%$ [30]. Similar to the USA, the risk might be even more elevated in adolescent sexual minority adolescent boys in China. In a large and nationally representative survey with more than 150,000 adolescent students from 506 high schools collected in 2014-15, one in five (21.6\%) adolescent boys who reported same-sex attraction and one in three $(34.7 \%)$ boys who reported both-sex attraction reported having experienced suicide ideation in the past year [43]. Further, this study found that $6.9 \%$ of boys reporting same-sex attraction and $12.2 \%$ of those reporting both-sex attraction attempted suicide in the past year, compared to $2.2 \%$ of their heterosexual peers [43].

Alcohol and Recreational Drug Use Injection drug use is relatively rare among Chinese MSM, yet use of alcohol and recreational drugs (i.e., club drugs), particularly before sex, is common. A recent meta-analysis summarizing 19 studies focused on alcohol use among Chinese MSM found a pooled prevalence of $23 \%$ for using alcohol more than once a week as well as for drinking prior to sex with male partners [83]. MSM who drank more than once per week were more likely to engage in drug use and sexual risk behaviors including condomless anal sex with men and trading sex for money [83]. Inhalant nitrites, also called poppers, are most popular recreational drugs reported among Chinese MSM [84]. For instance, a study sample of 3588 MSM from a HIV prevention intervention focusing on HIV testing in Beijing found $26.8 \%$ used poppers in the past month, which was associated with HIV infection and sexual risk behaviors (multiple male sex partnership and condomless anal intercourse) [85].

\section{The Mental Health of MSM Subpopulations}

Certain subgroups of MSM are found to be at heightened risk for mental health issues. "Money boys" refers to male sex workers in China who engage in sex with men for economic survival. Compared to the general MSM population, money boys have been found to experience more psychological distress. A study with MSM in Shanghai using respondentdriven sampling found that $70.0 \%$ money boys had depression symptoms compared to $46.1 \%$ of MSM not engaged in sex work [69]. Another study focusing on money boys in Shandong (northeast China) using respondent-driven sampling documented a similar rate of depression (68.1\%), with heterosexual-identified money boys more likely to suffer from psychological distress than their nonheterosexual counterparts [86]. Compared to MSM overall, money boys are more socioeconomically disadvantaged, at greater risk of sexual violence, substance abuse, and HIV and other STIs [86] - all could contribute to the high prevalence of depression in this group.

MSM who do not identify as gay (e.g., those who identify as bisexual, queer, unsure, or heterosexual) or those who also have sex with women may be even more vulnerable to mental 
health problems compared to their gay-identified peers. This is evident in the large national sample of Chinese adolescents in which boys who experienced both-sex attraction had far higher rates of suicide ideation and almost twice the rate of suicidal attempts than their male peers who report exclusively same-sex attraction [43]. A recent study conducted in Western and Southwestern cities of China using convenience sampling showed that $50.9 \%$ of men who have sex with men and women (MSMW) experienced depression compared to $35.2 \%$ of men who have sex with men only [41]. This is similar to research findings conducted in western countries suggesting that bisexual men (variously defined by identity, behavior, or attractions) are at greater risk for mental health issues compared to gay men $[1,87,88]$. The reason for such disparities in China has not been clearly identified, and as indicated by findings from western-based study samples [89], poor mental health experienced by bisexual people could be related to bisexual invisibility/erasure, experiences of bisexual-specific discrimination, biphobia in the gay community, and lack of support for bisexuality.

Despite many public health initiatives and evolved attitudes toward people living with HIV (PLWH) in China, HIV stigma is still pervasive in Chinese society [90, 91]. Thus, Chinese MSM living with HIV must adjust to living with a highly stigmatized health condition, which could further damage their mental health. A study in Southwest China found that $42.6 \%$ of MSM living with HIV suffered from moderateto-severe depression and $21.2 \%$ reported anxiety [47]; such high rates of psychological distress could adversely affect antiretroviral therapy adherence and quality of life [92-95]. For MSM living with HIV, the period following diagnosis and initiation of ART may be a particularly vulnerable time to distress and critical to successful care. A study in Beijing using convenience sampling from a HIV prevention and linkage-to-care trial found high rates of depression (39\%) and anxiety (42\%) among MSM newly diagnosed with HIV [61]. However, contrary to hypotheses, this study further found that depression and anxiety associated with early care initiation, suggesting that MSM who were aware of their high distress may be motivated to seek help during this period [61]. This could also be potentially due to ART initiation, and managing HIV care contributes to psychological distress. In another study using convenience sampling in Anhui Province among MSM living with HIV, one in three (31\%) endorsed suicide ideation in the past 6 months, which was associated with perceived distress in learning about one's HIV diagnosis, perceived HIV stigma and other mental health issues including depression and anxiety [67].

In summary, compared to the general population, MSM across regions in China are disproportionately affected by a range of mental health issues, including depression, anxiety, suicidal behaviors, and alcohol and substance dependence. Certain subgroups, such as those who engage in sex for money and those who are HIV positive and bisexual MSM are at an even heightened risk.

\section{Minority Stress as a Determinant of Mental Health Issues among Chinese MSM}

Derived from social psychological theories of stress, minority stress theory describes a process through which minority stressors negatively affect sexual minorities' mental health $[21,96]$. The model outlines a continuum of distal to proximal stressors specific to sexual orientation. Distal stressors may constitute objective, external stressful events such as prejudice and discrimination. Proximal stressors represent more internally oriented processes such as anticipation of prejudice events and internalization of stigmatizing attitudes such as internalized homophobia and concealment.

Given the primacy placed on the social context in generating minority stress [21], further highlighted in theories of structural stigma applied to sexual minorities [56], in this section, we first attend to the historic and cultural context of minority stress in China and provide a summary of distinct culturally related characteristics of minority stress concerning MSM. We then present a summary of research findings on the relationship between minority stress specific to sexual orientation/behavior, organized in terms of the distal-proximal continuum, and mental health in MSM. As minority stress theory has been called to be extended to HIV-related stress among MSM living with HIV [97], we also review findings on the relationship between HIV stigma and mental health among MSM in China.

Minority Stress for MSM in a Chinese Context To understand minority stress and its impact on Chinese MSM, it is important to situate their experiences in the relevant historic and sociocultural context in China. Societal attitudes toward same-sex behaviors among men in China have evolved over time. Generally speaking, pre-modern Chinese societies had fairly tolerant views toward MSM, often as long as these men fulfilled their family duty of marriage (with a woman) and procreation $[19,98]$. The traditional cultural values which emphasize heterosexual marriage, having children, and filial piety (i.e., obedience to parents) still persist today [20]. Further, in the late twentieth century, early viewpoints of western psychiatry were promulgated in China, leading homosexuality to be listed as a psychiatric disorder [19]. Although this diagnosis was removed from the Chinese Classification and Diagnostic Criteria of Mental Disorders in 2001, the view that same-sex attraction is pathological persisted, both among the public and the mental health professions [98]. Same-sex behaviors are not criminalized in China. However, same-sex marriage is illegal, and there are currently no laws prohibiting 
discrimination based on sexual orientation or gender identity/ expression [19, 99].

These historical and cultural characteristics give rise to distinct minority stress experiences among Chinese MSM. We characterize three salient patterns of a culturally embedded minority stress experience for MSM in China: (a) traditional family-oriented values of filial piety and fertility; (b) collectivistic culture that emphasizes norm conformity; and (c) identity concealment as a prevalent coping strategy.

First, traditional family-oriented values in filial piety and fertility are emphasized in Chinese men's social roles and duties, which shape the minority stress experience of MSM in China. Although minority stress experiences in the family context have been described among sexual minorities in western societies [100], this may be even more pronounced for Chinese MSM. In a national survey with more than 18,000 LGBT individuals in China, rejection from family was the most prevalent source of nonacceptance, experienced by $57.6 \%$ participants, compared to all other sources [101]. A study with LGB young adults in China found that a negative sexual identity was associated with parents' attitudes toward marriage, especially for young adults who placed higher value in filial piety [57]. Similarly, a study with Chinese MSM found that low levels of family support specific to sexual identity were linked to both internalized homophobia and psychological distress [60].

Second, experiences of minority stress for Chinese MSM occur in a collectivistic culture that emphasizes on social norms and norm conformity [20,60]. In a national survey with MSM, $96.9 \%$ of MSM reported being told that "homosexuality is not normal" by others [102]. As a largely collectivistic culture, Chinese society places high value in conforming to existing rules and norms. Another study with Chinese MSM found that endorsement of cultural value of norm conformity was associated with Chinese MSM's psychological distress, and internalized homophobia mediated the association between norm conformity and concealment of their sexual behavior and identity [60]. Being different from the heterosexual norm is regarded as "being abnormal," which in the Chinese context implies a problematic form of deviance and disease [103]. This view is still prevalent: in a recent survey with college students in China regarding their view of homosexuality, the agreement was strongest viewing it as a psychological disorder [104].

Third, in response to the prevalent heteronormative culture and conformity-focused values in China, Chinese MSM remain relatively hidden and largely conceal their sexual identity $[33,59]$. A national survey of Chinese MSM found that two thirds $(68.7 \%)$ had pretended to be heterosexual for social acceptance [102]. As a result of the strong familial and cultural pressure to marry and bear children, which has been intensified by the one-child policy from the 1980 s to 2010 s, a large proportion of MSM (as much as $80 \%$ by certain estimates) eventually marry a woman [105]. The cultural pressure to form a heterosexual marriage have resulted in a recent rise of "fake marriage markets" designed to help gay men and lesbians further conceal their sexual identity [105]. Qualitative analysis of minority stress in Chinese MSM suggests concealment as part of a coping process regarding to what degree MSM perform a heterosexual life [103]. This study further found that the concept of coming out was considered as "selfish" and "inconsiderate" to some MSM, insofar as the open declaration of one's personal sexual identity undermines cultural values of norm conformity, social harmony, and self-sacrifice [103].

Distal Sexual Minority Stress and Mental Health As noted, distal minority stress refers to external, objective prejudice events, such as discrimination and victimization due to one's sexual orientation. As Chinese MSM largely conceal their sexual orientation, exposure and experience of overt discrimination may be limited, especially in comparison with cultural contexts where openness about one's sexual identity is more common. In a national survey conducted with more than 700 MSM in China, 5.0\% reported experiences of physical violence, $3.7 \%$ had lost housing, and $5.2 \%$ lost a job in their lifetime due to sexual orientation [102]. Research findings are mixed regarding the role of discriminatory events in affecting the mental health of Chinese MSM. A longitudinal study in Beijing and a cross-sectional survey of a national sample of MSM in China reported no associations between experiences of discrimination (or enacted sexual identity stigma) and psychological distress, although both studies found discrimination to be associated with sexual risk behaviors [31, 102]. However, a study with MSM in Liaoning Province revealed that gay-related stressful life events in the past 3 months were associated with elevated risks of depression and suicidal behaviors $[53,73]$. Specifically, depression was most strongly linked to workplace-based gay-related stressful events (e.g., "trouble with boss or supervisor about same-sex behavior") [53]. In addition, "Getting in trouble with the police because of same-sex behavior" as a stressful event was associated with highest elevated risks of suicidal ideation, plan, and attempt, though the overall prevalence of this event was low [73].

In addition to discrimination, interpersonal-based victimization (e.g., bullying) could also lead to adverse psychological health. In a stratified and nationally representative sample of Chinese adolescents, $16.1 \%$ of LGB youth experienced school victimization (i.e., kicked, made fun of with sexual jokes, excluded from participation) in the past 30 days compared to $8.0 \%$ of heterosexual youth [106]. The study further found that school victimization mediated the association between sexual minority status and suicidal behavior, and this mediating effect was further moderated by peer and teacher relations such that those with poorer classmate or teacher relations experienced stronger victimization-associated suicidal 
behaviors [106]. Although not solely focused on MSM, this study supports research findings from western samples on experiences of bullying associated with suicidal behaviors among adolescents [107] and the association between sexual minority status and school victimization [108]. Similarly, another study with MSM in China found that experiencing interpersonal victimization (e.g., being rejected by others, humiliated, and bullied) were associated with psychological distress and internalized homophobia [60].

\section{Anticipated Stigma and Internalized Stigma and Mental}

Health As a result of living in a heteronormative and discriminative environment, sexual minorities may expect rejection, anticipate negative interactions, and internalize homophobic attitudes. Such processes are proximal to the self and maybe therefore likely to lead to psychological distress. A longitudinal study with MSM in Beijing found that anticipated MSM stigma affected both depression and anxiety through its association with increased avoidant and decreased social supportbased styles of coping with sexual identity-based stigma [32]. Thus, MSM who anticipate prejudice and rejection from others may attempt to avoid confronting problems and socially withdraw, which could further increase isolation and heighten distress. This finding provides support for the psychological mediation framework, which articulates that stigma creates elevations in maladaptive coping processes such as social avoidance, which in turn mediates the relationship between stigma and psychopathology [109].

Research on internalized homophobia in western societies finds that it plays a key role in shaping MSM's mental health $[27,110,111]$. However, research in China has yielded contradictory results: several studies, including both crosssectional and longitudinal, did not find internalized homophobia (or internalized sexual identity stigma) to be associated with or predict psychological distress [32, 60, 112]. A survey study of a large national sample of MSM in China found that minority stress process proximal to the self (i.e., internalized homonegativity, identity concealment) was not associated with psychological distress but that interpersonal-oriented identity concerns (i.e., acceptance concerns) and interpersonal victimization were associated with distress [60]. Similarly, a longitudinal study with MSM in Beijing did not find significant direct or indirect effects of internalized sexual identity stigma on depression and anxiety, but that anticipated stigma was predictive of these mental health issues [32]. Another study with Chinese LGB young adults did not find associations between proximal minority stress (i.e., internalized homophobia, self-concealment, and rejection sensitivity) and psychological maladjustment, yet social support, specifically perceived support from parents regarding one's sexuality, was associated with positive psychological outcomes [112]. On the other hand, two additional studies found that internalized homophobia was associated with poor psychological health.
One study, with MSM in Jiangsu Province, found that participants with high levels of depression had higher internalized homophobia compared to MSM with lower depression, and the group difference was significant [59]. Another study, with gay and bisexual men in Southwest China, categorized participants into two profiles based on their levels of internalized homophobia, and being in the "high internalized homophobia" group associated with more psychological distress and sexual compulsions [68]. However, both studies dichotomized the key variables (depression or internalized homophobia), which could reduce measurement sensitivity and increase the risk of a false positive and biased findings [113, 114].

Concealment and Mental Health Concealment can be conceptualized as a coping mechanism as well as a type of proximal minority stress $[21,22]$. Concealing a stigmatizing identity, although intended to protect oneself against discrimination and stigma, can nonetheless generate psychological distress that negatively affects one's mental health [22]. Across 157 studies largely derived from western samples, sexual identity disclosure among LGB individuals promotes social support and lowers psychological distress [115]. Among MSM in China, concealment has been found to be associated with higher internalized homophobia $[60,116]$. However, findings on the association between concealment and mental health of MSM in China have been mixed. Two large sample studies with Chinese MSM that measured concealment as a continuous variable did not find it to be significantly associated with mental health outcomes [60, 112]. By contrast, using betweengroup comparison methods based on binary categories (concealment versus non-concealment), studies have found that Chinese MSM who disclosed their sexual orientation had better psychological health compared to their counterparts who concealed their sexual orientation, supporting the positive role of identity disclosure $[15,55]$.

The lack of stronger evidence regarding the role of internalized homophobia and concealment in Chinese MSM's mental health does not imply that these experiences are irrelevant or unimportant to the overall wellness of Chinese MSM. In fact, these variables are associated with less frequent HIV testing, more frequent sexual risk behaviors, and more smoking behaviors $[31,33,74,102,117,118]$. However, their relationship to mental health warrants further inquiry and understanding, especially since various established psychosocial-based interventions informed by minority stress theory aim to reduce internalized homophobia and often promote disclosure as a pathway to lower distress and enhance well-being $[24,119,120]$. Features of the sociocultural and political environment both in the general public in China as well as the LGBTQ (lesbian, gay, bisexual, transgender, \& queer or questioning) community in China might explain the limited role of internalized homophobia and concealment in Chinese MSM's mental health. First, being in a collectivistic, 
relationship-oriented culture, Chinese MSM's psychological health may be more influenced by processes that are more relational compared to their western counterparts. This has been supported by cross-cultural research with the general Chinese population [121] and future comparison research focused on MSM is needed. In a related vein, internalized homophobia and concealment may influence mental health through relational-oriented (rather than self-oriented) processes such as anticipated stigma, social isolation, and avoidance coping $[32,60]$. Second, since Chinese MSM largely conceal their sexuality, sexual identity may not be particularly salient to participants' everyday life or integrated into their sense of self, especially in a culture where the construction of self is relatively more context-based $[122,123]$. This could impact the magnitude of influence that internalized homophobia has on the psychological health of MSM. Third, there is less pressure for "coming out" in the LGBT community in China compared to western countries due to the community's understanding of the larger sociocultural environment as well as cultural differences in the perception of "coming out" [103, 124]. Thus, concealment may be considered as a culturally appropriate and adaptive coping strategy. Further, due to MSM community's hiddenness, coming out may not necessarily facilitate MSM to easily access and gain support from a larger MSM community.

HIV-Related Stigma and Mental Health Similar to a sexual identity and behavior, HIV infection is also a concealable and stigmatizing status. It has been argued that the minority stress framework concerning MSM should be extended to include HIV status, as suggested by empirical evidence noting the deleterious effect of HIV stigma as a stressor for MSM living with HIV [97, 125, 126]. Among Chinese MSM living with HIV, studies highlight the detrimental influences of enacted, perceived, and internalized HIV stigma. A study in Chengdu City (Southwest China) with MSM living with HIV found enacted HIV stigma to be associated with depression [47]. In another study of MSM living with HIV in Anhui (Northcentral China), perceived HIV stigma was associated with suicidal ideation in the past 6 months [67]. In yet another study among MSM living with HIV in Nanjing (Central China), perceived HIV stigma was associated with depression, and this relationship was mediated through poorer social support and self-esteem [70]. This suggests that perceived HIV stigma might affect MSM's self-evaluation and social support, which then impacts their mental health. A study with MSM in China newly diagnosed with HIV measured four types of HIV stigma (enacted, felt, vicarious, and internalized) and found that internalized HIV stigma to most strongly correlate with depression [62]. Two other studies also found internalized HIV stigma to strongly predict mental health $[49,71]$. A study with MSM living with HIV in Hong Kong measured both perceived and internalized HIV stigma and specifically focused on perceived HIV stigma within the MSM community [38]. This study revealed an association between perceived HIV stigma within the community and psychological distress, and this relationship was mediated by internalized HIV stigma, HIV disclosure concerns, and negative reactions toward facing stigmatizing attitudes from HIV-negative MSM [38]. Findings from this study reveal the importance of MSM's experience of HIV stigma within their community, which can create further social isolation and degraded self-worth for MSM living with HIV. For HIV-negative or serostatusunknown MSM, anticipatory HIV stigma can also be detrimental to behavioral health. A study of more than 2000 MSM and transgender individuals in China found that anticipated HIV stigma was associated with less disclosure of one's sexual orientation to healthcare provider as well as likelihood of not being tested for HIV [127].

\section{The Need for LGBT-Affirmative Care in China}

The field of counseling and psychotherapy services is new and underdeveloped field in China for the general population overall. Awareness of mental illness is still lacking in the general public, and stigma for seeking professional psychological services remains prevalent, resulting in only $10 \%$ of those with a psychiatric illness that actually seek care [128]. It is estimated that approximately 173 million people in China suffer from mental illnesses, with only 40,000 certified counselors practicing on an either full- or part-time basis [128, 129]. In the past decade, the government responded to the high demand for mental health by issuing a certification process for counselors with relatively low standards (e.g., no requirement for a graduate degree or for supervision or practicum experience), which has resulted in mixed quality of training among providers [130]. A national survey of practitioners found that $70 \%$ had a bachelor's level education or lower degree, and only $36.4 \%$ had majored in psychology [130].

The lack of sufficient professional training in China, along with the history of pathologizing same-sex behaviors, have resulted in prevalent malpractice in mental health services for LGBT clients. Conversion therapy has been advertised by many clinics, with an emphasis on aversion-based treatment [131]. A 2014 lawsuit in Chongqing against conversion therapy, which produced a ruling in favor of the patient, has brought this issue to the public eye [131]. A 2015 national survey with more than 1000 counselors in China, however, revealed many concerning issues: despite $88.6 \%$ counselors reporting that they "accept homosexuality," only $67.5 \%$ endorsed acceptance for bisexuality and $41.7 \%$ endorsed acceptance for transgender people [132]. More concerningly, 86.9\% of counselors reported that sexual orientation can be changed. From a list of possible reasons for the development of nonheterosexual sexual orientation, "family-related influence" was 
the most commonly endorsed by counselors (87.7\%), followed by sexual experiences (72.5\%) and genetics (62.0\%) [132]. This may not be surprising given that psychoanalysis was among the first western psychotherapy approaches introduced in China in the 1980s, and early psychoanalytic views of sexuality has gained popularity in the profession [133]. In this survey, more than a third of counselors $(35.3 \%)$ viewed homosexuality as a psychological illness [132]. The pathological view of same-sex behaviors, along with the belief that samesex attraction is due to environmental factors, may explain the common endorsement of conversion therapy among professionals. More than a third of counselors (36\%) reported support for conversion therapy, and among them, $86 \%$ reported that they had practiced conversion therapy [132]. Adverse experiences in therapy due to professionals' discriminatory views and behaviors, including conversion therapy, can further damage LGBT clients' mental health $[134,135]$. A qualitative inquiry with MSM in Hong Kong regarding their experiences in counseling suggests that many MSM experienced overt judgments from counselors about their sexual orientation (e.g., counselors asking the client to stop talking about it) as well as their sexuality being dismissed and pathologized [136], discouraging them from seeking care in the future.

In addition to counselor-related factors, minority stress also affects MSM's help-seeking behaviors. In a sample of MSM in Hong Kong who reported having mental health issues in the past year, those who reported higher internalized homophobia and perceived public stigma toward seeking professional psychological help were less willing to seek help, while those who perceived more empathy from mental health professions had higher levels of intention to seek service [58]. On the other hand, counseling can facilitate disclosure: MSM who reported ever receiving counseling were more likely to disclose their same-sex behavior with their family [58]. Minority stress, mental health stigma, and the prevalent pathological view of same-sex behaviors (along with the practice of conversion therapy) by many mental health professionals can result in maintenance of psychological distress, underutilization of mental health services, and ineffective treatment for MSM in China. Therefore, it is critical to train counselors to provide competent, culturally relevant, LGBT-affirmative care for MSM in China.

Despite the high need to address minority stress and mental health among MSM in China, we could only locate one intervention that focused on the psychological health of Chinese MSM, specifically MSM newly diagnosed with HIV [137]. This culturally tailored, brief 3-session CBT skills-based pilot study with ten MSM recently diagnosed with HIV was found to improve coping with their HIV diagnosis, reduce depression, and increase perceived social support [137]. Interventions in the USA and other global regions (e.g., Canada, India, Thailand) that focused on stigma reduction and improving psychological health of MSM have shown to be effective in reducing minority stress and psychological distress, promoting disclosure, and increasing safe sex behaviors [24, 138-140]. More interventions in China focusing on minority stress and mental health of MSM are needed to address mental health and treatment disparities.

\section{Addressing Minority Stress and Mental Health in Chinese MSM: Recommendations for Intervention Development}

Current HIV-related efforts with MSM in China mostly focus on educational and behavioral aspects of HIV prevention and treatment, such as HIV education and condom distribution. While important and necessary, HIV prevention and treatment efforts with MSM that solely focus on behaviors are likely to have limited effects, if factors leading to HIV risk such as sexual identity stigma and depressive symptoms are not adequately addressed $[12,141]$. More psychosocial-focused interventions are needed to address barriers to health behaviors for the many MSM in China who are affected by minority stress and psychological distress. If designed and delivered properly, such interventions may successfully reduce the burden of HIV among MSM in China while enhancing their mental health. Based on findings summarized in this review, we provide the following suggestions and recommended considerations in the development of such interventions.

First, interventions that aim to reduce minority stress and associated distress among Chinese MSM may benefit from a culturally relevant conceptualization of minority stress and mental health $[60,103]$. As illustrated in a grounded theory analysis where researchers proposed a culturally-responsive model of minority stress theory for Chinese MSM, important cultural constructs including heterosexual marriage, procreation, and filial piety organize the heteronormative cultural context in which they live [103]. Collectivistic cultural values, particularly norm conformity, can further elevate distress related to minority stress [60]. Though individual-level interventions are not designed to change the larger sociocultural norms, they can help MSM develop awareness of their environment, produce insight into how environmental factors affect their mood and behaviors, and enhance skills for coping with minority stress and navigating through a non-affirming environment. When considering culturally relevant intervention, it is also important to pay attention to nuances regarding what may be considered as healthy versus maladaptive coping in the cultural context. For example, disclosure could bring the benefits of social support and decrease the psychological and cognitive burden associated with concealment, yet concealment can also be an adaptive (and widely employed) strategy for Chinese MSM who live in non-affirming environments [103]. Further, given the relational and collectivistic cultural context surrounding Chinese MSM, interventions that target 
proximal types of minority stressors (i.e., internalized homophobia) may want to offer a relational-informed conceptualization to make it culturally congruent and to help MSM bring enhanced skills (e.g., assertiveness, self-advocacy) and selfworth into their social and romantic relationships $[60,103]$. In this regard, an affirming, supportive, and validating therapeutic stance is also critical to provide a relational context where MSM can experience corrective emotions and cognitions regarding their sexual identity.

Second, individual-level interventions such as psychotherapy are needed to address the psychosocial challenges facing MSM and serve as an alternative to conversion therapy. In particular, interventions need to target a wide array of commonly experienced mental health issues by Chinese MSM such as depression, anxiety, suicidal behaviors, and alcohol dependence. Given the important role of minority stress in shaping the experience of mental health issues by Chinese MSM, targeting common pathways through which minority stress generates distress can be a promising, transdiagnostic approach to engage and empower MSM [142]. For example, such treatments may help to normalize and "de-pathologize" MSM's experiences of same-sex attractions and behaviors, enhance self-acceptance, promote supportive relationships, and facilitate skills development to help them cope with minority stress at familial, community, and cultural levels.

Third, group-based interventions may be helpful for MSM to decrease isolation, build community, and learn from others' experiences and strategies of coping with familial and culturally related minority stressors. This may serve as a helpful bridge to mental health services for MSM who experience stigma related to seeking professional psychological help and/or are cautious about counselors' potential judgmental and non-accepting view of their sexuality. Family-based interventions may also be beneficial to reduce stigma in MSM's familial environment. For instance, an attachment-focused family therapy in Israel has been found to be effective for resolving conflict between non-accepting parents and sexual minority children [143]. Adaptations of such an intervention could be promising for MSM in China.

Fourth, intervention development and implementation efforts are needed to train mental health professionals in China to provide culturally relevant, competent, LGBT-affirmative therapy. Such efforts have been made by nonprofit community organizations such as the Beijing LGBT Center and other psychology training institutions in China as continued education programs. While these trainings have not been empirically evaluated, anecdotal evidence and evidence from other high-stigma countries [144] suggest they can be a promising way to facilitate counselors to develop an affirmative conceptualization of their sexual and gender minority clients as well as raise awareness regarding the harms of conversion therapy. Barriers to achieve more effective training, per personal observation through providing such education programs in
China, may include (a) reaching a relatively limited pool of counselors who are already LGBT affirmative; (b) counselors' varied training backgrounds and lacking basic counseling skills training and supervision experiences; and (c) the lack of continued support and supervision for counselors following training and education programs. More systematic efforts, such as guidelines for LGBT-affirmative therapy, prohibitive regulations on conversion therapy, and competency courses as part of the requirement for counselor training, could bring fruitful results.

Fifth, given the harmful effect of minority stress on the well-being of MSM, this population requires interventions that address the larger, societal forms of prejudice against sexual minorities and that promote LGBT-affirmative policies in order to diminish this ultimate source of distress. Given the structural and cultural barriers in China, intervention developers need to work collaboratively with the MSM community to skillfully navigate the complex sociocultural and political environment in China. One strategic position for achieving this goal would be to highlight the damaging role of mental health issues in HIV risk of MSM as well as to the need to address this public health concern through psychosocial interventions and advocacy efforts.

\section{Conclusions}

In conclusion, Chinese MSM experience elevated risks of various mental health conditions, which are linked to minority stress. Addressing minority stress in a culturally relevant way is imperative to reduce stigma and promote mental health, and this effort is needed on individual and community levels, engaging both MSM and their providers. Additional research, ideally using populationbased sampling, is needed to further identity the prevalence of mental health problems among Chinese MSM. Additional research is also needed to delineate the mechanisms through which minority stress compromises mental health among Chinese MSM. Finally, intervention research is needed to evaluate a minority stress-informed approach for reducing minority stress and other relevant stigmas (e.g., HIV stigma) and their adverse psychological and behavioral health impact. If shown to be effective, this approach may have implications for prevention and treatment to the syndemic risks and achieve optimal health outcomes among MSM in China and broader global regions where cultural nuances shape manifestations of stigma and mental health.

Acknowledgments Effort by the first author was supported by the National Institute of Mental Health (T32MH078788) and the Providence/Boston Center for AIDS Research (P30AI042853). 


\section{Compliance with Ethical Standards}

Conflict of Interest None of the authors have any conflict of interest to declare.

Human and Animal Rights and Informed Consent This article does not contain any studies with human or animal subjects performed by any of the authors.

\section{References}

1. Bostwick WB, Boyd CJ, Hughes TL, McCabe SE. Dimensions of sexual orientation and the prevalence of mood and anxiety disorders in the United States. Am J Public Health. 2010;100(3):468 75.

2. Stoloff K, Joska JA, Feast D, de Swardt G, Hugo J, Struthers H, et al. A description of common mental disorders in men who have sex with men (MSM) referred for assessment and intervention at an MSM clinic in Cape Town South Africa. AIDS Behav. 2013;17(S1):77-81.

3. Beyrer C, Baral SD, Van Griensven F, Goodreau SM, Chariyalertsak S, Wirtz AL, et al. Global epidemiology of HIV infection in men who have sex with men. Lancet. 2012;380(9839):367-77.

4. Luo Z, Feng T, Fu H, Yang T. Lifetime prevalence of suicidal ideation among men who have sex with men: A meta-analysis. BMC Psychiatry. 2017;17(1):1-9.

5. Sivasubramanian M, Mimiaga MJ, Mayer KH, Anand VR, Johnson CV, Prabhugate P, et al. Suicidality, clinical depression, and anxiety disorders are highly prevalent in men who have sex with men in Mumbai, India: Findings from a community-recruited sample. Psychol Health Med. 2011;16(4):450-62.

6. Tomori C, McFall AM, Srikrishnan AK, Mehta SH, Solomon SS, Anand $\mathrm{S}$, et al. Diverse rates of depression among men who have sex with men (MSM) across India: Insights from a multi-site mixed method study. AIDS Behav. 2016;20(2):304-16.

7. Kerridge BT, Pickering RP, Saha TD, Ruan WJ, Chou SP, Zhang $\mathrm{H}$, et al. Prevalence, sociodemographic correlates and DSM-5 substance use disorders and other psychiatric disorders among sexual minorities in the United States. Drug Alcohol Depend. 2017;170:82-92.

8. King M, Semlyen J, Tai SS, Killaspy H, Osborn D, Popelyuk D, et al. A systematic review of mental disorder, suicide, and deliberate self harm in lesbian, gay and bisexual people. BMC Psychiatry. 2008;8:1-17.

9. McCabe SE, Hughes TL, Bostwick WB, West BT, Boyd CJ. Sexual orientation, substance use disorders and substance dependence in the United States. Addiction. 2009;104(8):1333-45.

10. Mustanski B, Andrews R, Herrick A, Stall R, Schnarrs PW. A syndemic of psychosocial health disparities and associations with risk for attempting suicide among young sexual minority men. Am J Public Health. 2014;104(2):287-94.

11. Tsai AC, Burns BFO. Syndemics of psychosocial problems and HIV risk: A systematic review of empirical tests of the disease interaction concept. Soc Sci Med. 2015;139:26-35.

12. Safren SA, Reisner SL, Herrick A, Mimiaga MJ, Stall R. Mental health and HIV risk in men who have sex with men. J Acquir Immune Defic Syndr. 2010;55(Suppl 2):S74-7.

13. National Health and Family Planning Commission of China. 2015 China AIDS Response Progress Report. In: Global AIDS Response Progress Reporting. 2015. p. 1-26.
14. Zheng S. The growing threat of China's HIV epidemic. Lancet Public Health. 2018;3(7):e311.

15. Liu J, Yi Z, Zhao Y, Qu B, Zhu Y. The psychological health and associated factors of men who have sex with men in China: A cross-sectional survey. PLoS One. 2018;13(5):1-14.

16. Pan X, Li R, Ma Q, Wang H, Jiang T, He L, et al. Sexual risk behaviour, sexual victimization, substance use and other factors related to depression in men who have sex with men in Wenzhou, China: A cross-sectional study. BMJ Open. 2018;8(4):1-10.

17. Mu H, Li Y, Liu L, Na J, Yu L, Bi X, et al. Prevalence and risk factors for lifetime suicide ideation, plan and attempt in Chinese men who have sex with men. BMC Psychiatry. 2016;16(1):1-11.

18. Yu L, Jiang C, Na J, Li N, Diao W, Gu Y, Zhao L, Zou Y, Chen Y, Liu L, Mu H, Liu Y, Yu L, Yang X, Pan G. Elevated 12-month and lifetime prevalence and comorbidity rates of mood, anxiety, and alcohol use disorders in Chinese men who have sex with men. PLoS One. 2013;8(4).

19. Zhang B, Chu Q. MSM and HIV/AIDS in China. Cell Res. 2010;15:858-64.

20. Steward WT, Miège P, Choi K-H. Charting a moral life: The influence of stigma and filial duties on marital decisions among Chinese men who have sex with men. PLoS One. 2013;8(8): e71778.

21. Meyer IH. Prejudice, social stress, and mental health in lesbian, gay, and bisexual populations: Conceptual issues and research evidence. Psychol Bull. 2003;129(5):674-97.

22. Pachankis JE. The psychological implications of concealing a stigma: A cognitive-affective-behavioral model. Psychol Bull. 2007;133(2):328-45.

23. Goldbach JT, Tanner-Smith EE, Bagwell M, Dunlap S. Minority stress and substance use in sexual minority adolescents: A metaanalysis. Prev Sci. 2014;15(3):350-63.

24. Pachankis JE, Hatzenbuehler ML, Rendina HJ, Safren SA, Parsons JT. LGB-affirmative cognitive-behavioral therapy for young adult gay and bisexual men: A randomized controlled trial of a transdiagnostic minority stress approach. J Consult Clin Psychol. 2015;83(5):875-89.

25. Hatzenbuehler ML, Nolen-Hoeksema S, Erickson SJ. Minority stress predictors of HIV risk behavior, substance use, and depressive symptoms: Results from a prospective study of bereaved gay men. Health Psychol. 2008;27(4):455-62.

26. Pachankis JE, Hatzenbuehler ML, Hickson F, Weatherburn P, Berg RC, Marcus U, et al. Hidden from health: Structural stigma, sexual orientation concealment, and HIV across 38 countries in the European MSM Internet Survey. AIDS. 2015;29(10):1239-46.

27. Pachankis JE, Sullivan TJ, Feinstein BA, Newcomb ME. Young adult gay and bisexual men's stigma experiences and mental health: An 8-year longitudinal study. Dev Psychol. 2018;54(7): 1381-93.

28. Storholm ED, Satre DD, Kapadia F, Halkitis PN. Depression, Compulsive Sexual Behavior, and Sexual Risk-Taking Among Urban Young Gay and Bisexual Men: The P18 Cohort Study. Arch Sex Behav. 2016;45(6):1431-41.

29. Guo Y, Li X, Stanton B. HIV-related behavioral studies of men who have sex with men in China: A systematic review and recommendations for future research. AIDS Behav. 2011;15(3):52134.

30. Chen H, Li Y, Wang L, Zhang B. Causes of suicidal behaviors in men who have sex with men in China: A national questionnaire survey. BMC Public Health. 2015;15(1):15-8.

31. Choi K-H, Steward WT, Miege P, Gregorich SE. Unpacking the influence of sexual stigma on HIV risk: Results from a prospective study of men who have sex with men in Beijing China. J Acquir Immune Defic Syndr. 2017;74(2):e38-44.

32. Choi K-H, Steward WT, Miège P, Hudes E, Gregorich SE. Sexual stigma, coping styles, and psychological distress: A longitudinal 
study of men who have sex with men in Beijing China. Arch Sex Behav. 2016;45(6):1483-91.

33. Zhao Y, Ma Y, Chen R, Li F, Qin X, Hu Z. Non-disclosure of sexual orientation to parents associated with sexual risk behaviors among gay and bisexual MSM in China. AIDS Behav. 2016;20(1):193-203

34. Mirowsky J, Ross CE. Age and depression. J Health Soc Behav. 1992;33(3):187-205

35. Derogatis LM. Brief symptom inventory: An introductory report. Psychol Rep. 1983;13:595-605.

36. Steward WT, Herek GM, Ramakrishna J, Bharat S, Chandy S, Wrubel J, et al. HIV-related stigma: Adapting a theoretical framework for use in India. Soc Sci Med. 2008;67(8):1225-35.

37. Wolfe WR, Weiser SD, Leiter K, Steward WT, Percy-de Korte F, Phaladze N, et al. The impact of universal access to antiretroviral therapy on HIV stigma in Botswana. Am J Public Health. 2008;98(10):1865-71.

38. Chong ESK, Mak WWS, Tam TCY, Zhu C, Chung RWY. Impact of perceived HIV stigma within men who have sex with men community on mental health of seropositive MSM. AIDS Care. 2017;29(1):118-24.

39. Berwick DM, Murphy JM, Goldman PA, Ware JEJ, Barsky AJ, Weinstein MC. Performance of a five-item mental health screening test. Med Care 29 AD. 2:169-76.

40. Mak WWS, Cheung RYM. Self-stigma among concealable minorities in Hong Kong: Conceptualization and unified measurement. Am J Orthop. 2010;80(2):267-81.

41. Hu Y, Zhong XN, Peng B, Zhang Y, Liang H, Dai JH, et al. Comparison of depression and anxiety between HIV-negative men who have sex with men and women (MSMW) and men who have sex with men only (MSMO): A cross-sectional study in Western China. BMJ Open. 2019;9(1):1-11.

42. Zhang Y, Liu R, Li G, Mao S, Yuan Y. The reliability and validity of a Chinese-version Short Health Anxiety Inventory: An investigation of university students. Neuropsychiatr Dis Treat. 2015;11: 1739-47.

43. Huang Y, Li P, Guo L, Gao X, Xu Y, Huang G, et al. Sexual minority status and suicidal behaviour among Chinese adolescents: A nationally representative cross-sectional study. BMJ Open. 2018;8(8):1-9.

44. Ibragimov U, Harnisch JA, Nehl EJ, He N, Zheng T, Ding Y, et al. Estimating self-reported sex practices, drug use, depression, and intimate partner violence among MSM in China: A comparison of three recruitment methods. AIDS Care. 2017;29(1):125-31.

45. Li R, Cai Y, Wang Y, Gan F, Shi R. Psychological pathway to suicidal ideation among men who have sex with men in Shanghai, China: A structural equation model. J Psychiatr Res. 2016;83: 203-10.

46. Li R, Cai Y, Wang Y, Sun Z, Zhu C, Tian Y, et al. Psychosocial syndemic associated with increased suicidal ideation among men who have sex with men in Shanghai China. Health Psychol. 2016;35(2):148-56.

47. Li J, Mo PKH, Kahler CW, Lau JTF, Du M, Dai Y, et al. Prevalence and associated factors of depressive and anxiety symptoms among HIV-infected men who have sex with men in China. AIDS Care. 2016;28(4):465-70.

48. Spitzer RL, Kroenke K, Williams JB, Lowe B. A brief measure for assessing generalized anxiety disorder: The GAD-7. Arch Intern Med. 2006;166(10):1092-7.

49. Li J, Mo PKH, Wu AMS, Lau JTF. Roles of self-stigma, social support, and positive and negative affects as determinants of depressive symptoms among HIV infected men who have sex with men in China. AIDS Behav. 2017;21(1):261-73.

50. Watson D, Clark LA, Tellege A. Development and validation of brief measures of positive and negative affect: The PANAS scales. J Pers Soc Psychol. 1988;54:1063-70.
51. Liu Y, Ruan Y, Strauss SM, Yin L, Liu H, Amico KR, et al. Alcohol misuse, risky sexual behaviors, and HIV or syphilis infections among Chinese men who have sex with men. Drug Alcohol Depend. 2016;168:239-46.

52. Saunders JB, Aasland OG, Babor TF, de la Fuente JR, Grant M. Development of the alcohol use disorders identification test (AUDIT): WHO collaborative project on early detection of persons with harmful alchol consumption-II. Addiction. 1993;88: 791-904.

53. Liu Y, Jiang C, Li S, Gu Y, Zhou Y, An X, et al. Association of recent gay-related stressful events with depressive symptoms in Chinese men who have sex with men. BMC Psychiatry. 2018;18(1):217.

54. Rosario M, Schrimshaw EW, Hunter J, Gwadz M. Gay-related stress and emotional distress among gay and bisexual youths: A longitudinal examination. J Consult Clin Psychol. 2002;70(4): 967-75.

55. Liu X, Jiang D, Chen X, Tan A, Hou Y, He M, et al. Mental health status and associated contributing factors among gay men in China. Int J Environ Res Public Health. 2018;15(6):1065.

56. Hatzenbuehler ML. Structural stigma and health inequalities: Research evidence and implications for psychological science. Am Psychol. 2016;71(8):742-51.

57. Hu X, Wang Y. LGB Identity among young Chinese: The influence of traditional culture. J Homosex. 2013 May 1;60(5):667-84.

58. Mo PKH, Lau JTF, Lau MMC, Kim Y. Mental health service utilization among men who have sex with men who are at risk of mental health problems in Hong Kong: Prevalence and associated factors. Psychol Men Masculinity. 2018;19(3):392-406.

59. Su X, Zhou AN, Li J, Shi LE, Huan X, Yan H, et al. Depression, loneliness, and sexual risk-taking among HIV-negative/unknown men who have sex with men in China. Arch Sex Behav. 2017;47(7):1959-68 1-10.

60. Sun S, Tarantino N, Whiteley L, Pachankis JE, Hoyt WT, Operario D, Brown LK. Cultural context matters: Testing the minority stress model among Chinese sexual minority men. J Couns Psychl. 2020; Under Review.

61. Tao J, Vermund SH, Lu H, Ruan Y, Shepherd BE, Kipp AM, et al. Impact of depression and anxiety on initiation of antiretroviral therapy among men who have sex with men with newly diagnosed HIV infections in China. AIDS Patient Care STDs. 2017;31(2): 96-104.

62. Tao J, Wang L, Kipp AM, Qian HZ, Yin L, Ruan Y, et al. Relationship of stigma and depression among newly HIVdiagnosed Chinese men who have sex with men. AIDS Behav. 2017;21(1):292-9.

63. Wang Z, Zhao X, Zhang Z, Luo M, Shen Q, Dong Y, et al. Cooccuring psychosocial problems with multiple sexual partners among men who have sex with men in Shanghai, China: A syndemic approach. J Sex Res. 2018;55(7):892-901.

64. Wang Y, Wang Z, Jia M, Liang A, Yuan D, Sun Z, et al. Association between a syndemic of psychosocial problems and unprotected anal intercourse among men who have sex with men in Shanghai China. BMC Infect Dis. 2017;17(1):1-9.

65. Wang Y, Dong $\mathrm{M}$, Zhang Q, Xu D, Zhao J, Ng CH, et al. Suicidality and clinical correlates in Chinese men who have sex with men (MSM) with HIV infection. Psychol Health Med. 2019;24(2):137-43.

66. Wang N, Wang S, Qian HZ, Ruan Y, Amico KR, Vermund SH, et al. Negative associations between general self-efficacy and anxiety/depression among newly HIV-diagnosed men who have sex with men in Beijing, China. AIDS Care. 2019;31(5):629-35.

67. Wu Y, Yang H-Y, Wang J, Yao H, Zhao X, Chen J, et al. Prevalence of suicidal ideation and associated factors among HIV-positive MSM in Anhui. China Int J STD AIDS. 2015;26(7):496-503. 
68. Xu W, Zheng L, Xu Y, Zheng Y. Internalized homophobia, mental health, sexual behaviors, and outness of gay/bisexual men from Southwest China. Int J Equity Health. 2017;16(1):1-10.

69. Yan H, Wong FY, Zheng T, Ning Z, Ding Y, Nehl EJ, et al. Social support and depressive symptoms among "money" boys and general men who have sex with men in Shanghai, China. Sex Health. 2014;11(3):285.

70. Yan H, Li X, Li J, Wang W, Yang Y, Yao X, et al. Association between perceived HIV stigma, social support, resilience, selfesteem, and depressive symptoms among HIV-positive men who have sex with men (MSM) in Nanjing. China AIDS Care. 2019;31(9):1069-76 0(0):1-8.

71. Yang X, Mak WWS, Ho CYY, Chidgey A. Self-in-love versus self-in-stigma: implications of relationship quality and love attitudes on self-stigma and mental health among HIV-positive men having sex with men. AIDS Care. 2017;29(1):132-6.

72. Ye Z, Chen L, Lin D, Wang SX. The relationship between posttraumatic stress disorder symptoms and posttraumatic growth among HIV-Infected men who have sex with men in Beijing, China: The mediating roles of coping strategies. Front Psychol. 2018;9(September):1-9.

73. Yu L, Li Y, Liu L, Li S, Na J, An X, Zhou Y, Gu Y, Bi X, Mu H, Zhang R, Dong W, Pan G. Association of recent gay-related stressful events and emotional distress with suicidal behaviors over 12 months in Chinese men who have sex with men. Asia-Pacific Psychiatry. 2018;10(1).

74. Yu F, Nehl EJ, Zheng T, He N, Berg CJ, Lemieux AF, et al. A Syndemic including cigarette smoking and sexual risk behaviors among a sample of MSM in Shanghai, China. Drug Alcohol Depend. 2013;132(0):265-70.

75. Zhang H, Wong WCW, Ip P, Fan S, Yip PSF. Health status and risk behaviors of sexual minorities among Chinese adolescents: A School-based survey. J Homosex. 2017;64(3):382-96.

76. Zhu Y, Liu J, Chen Y, Zhang R, Qu B. The relation between mental health, homosexual stigma, childhood abuse, community engagement, and unprotected anal intercourse among MSM in China. Sci Rep. 2018;8(1):1-7.

77. Huang Y, Wang Y, Wang H, Liu Z, Yu X, Yan J, et al. Prevalence of mental disorders in China: a cross-sectional epidemiological study. Lancet Psychiatry. 2019;6(3):211-24.

78. Burnham KE, Cruess DG, Kalichman MO, Grebler T, Cherry C, Kalichman SC. Trauma symptoms, internalized stigma, social support, and sexual risk behavior among HIV-positive gay and bisexual MSM who have sought sex parnters online. AIDS Care. 2016;28(3):347-53.

79. Reisner SL, Mimiaga MJ, Safren SA, Mayer KH. Stressful or traumatic life events, post-traumatic stress disorder (PTSD) symptoms, and HIV sexual risk taking among men who have sex with men. AIDS Care - Psychol Socio-Medical Asp AIDS/HIV. 2009;21(12):1481-9.

80. Choi KW, Batchelder AW, Ehlinger PP, Safren SA, O'Cleirigh C. Applying network analysis to psychological comorbidity and health behavior: Depression, PTSD, and sexual risk in sexual minority men with trauma histories. J Counsult Clin Psychol. 2017;85(12): 1158-70.

81. Hottes TS, Bogaert L, Rhodes AE, Brennan DJ, Gesink D. Lifetime prevalence of suicide attempts among sexual minority adults by study sampling strategies: A systematic review and meta-analysis. Am J Public Health. 2016;106(5):e1-12.

82. Cao X-L, Zhong B-L, Xiang Y-T, Ungvari GS, Lai KYC, Chiu HFK, et al. Prevalence of suicidal ideation and suicide attempts in the general population of China: A meta-analysis. Int J Psychiatry Med. 2015;49(4):296-308.

83. Liu Y, Qian H-Z, Ruan Y, Yin L, Ma J, Dahiya K, et al. Alcohol use among Chinese men who have sex with men: An epidemiological survey and meta-analysis. Biomed Res Int. 2014:1-11.
84. Nehl EJ, He N, Lin L, Zheng T, Harnisch JA, Ding Y, et al. Drug use and sexual behaviors among MSM in China. Subst Use Misuse. 2015;50(1):123-36.

85. Zhang H, Teng T, Lu H, Zhao Y, Liu H, Yin L, et al. Poppers use and risky sexual behaviors among men who have sex with men in Beijing, China. Drug Alcohol Depend. 2016;160:42-8.

86. Tao X, Gai R, Zhang N, Zheng W, Zhang X, Xu A, et al. HIV infection and mental health of "money boys": A pilot study in Shandong Province, China. Southeast Asian J Trop Med Public Heal. 2010;41(2):358-68.

87. Conron KJ, Mimiaga MJ, Landers SJ. A population-based study of sexual orientation identity and gender differences in adult health. Am J Public Health. 2010;100(10):1953-60.

88. Mills TC, Paul J, Stall R, Pollack L, Canchola J, Chang YJ, et al. Distress and depression in men who have sex with men: The Urban Men's Health Study. Am J Psychiatry. 2004;161:278-85.

89. Ross LE, Salway T, Tarasoff LA, MacKay JM, Hawkins BW, Fehr CP. Prevalence of depression and anxiety among bisexual people compared to gay, lesbian, and heterosexual individuals: A systematic review and meta-analysis. J Sex Res. 2018;55(4-5):435-56.

90. Zhang C, Li X, Liu Y, Qiao S, Zhang L, Zhou Y, et al. Stigma against People Living with HIV/AIDS in China: Does the Route of Infection Matter? PLoS One [Internet]. 2016;11(3):e0151078.

91. Li L, Wu Z, Liang LJ, Lin C, Guan J, Jia M, et al. Reducing HIVrelated stigma in health care settings: A randomized controlled trial in China. Am J Public Health. 2013;103(2):286-92.

92. Uthman OA, Magidson JF, Safren SA, Nachega JB. Depression and adherence to antiretroviral therapy in low-, middle- and highincome countries: A systematic review and meta-analysis. Curr HIV/AIDS Rep. 2014;11(3):291-307.

93. Brandt C, Zvolensky MJ, Woods SP, Gonzalez A, Safren SA, O'Cleirigh CM. Anxiety symptoms and disorders among adults living with HIV and AIDS: A critical review and integrative synthesis of the empirical literature. Clin Psychol Rev [Internet]. 2017:51:164-84.

94. Rubin LH, Maki PM. HIV, Depression, and Cognitive Impairment in the Era of Effective Antiretroviral Therapy. Curr HIV/AIDS Rep. 2019;16(1):82-95.

95. Rueda S, Mitra S, Chen S, Gogolishvili D, Globerman J, Chambers L, et al. Examining the associations between HIVrelated stigma and health outcomes in people living with HIV/ AIDS: a series of meta-analyses. BMJ Open. 2016;6(7):e011453.

96. Meyer IH. Prejudice as stress: Conceptual and measurement problems. Am J Public Health. 2003;93(2):262-5.

97. Rendina HJ, Gamarel KE, Pachankis JE, Ventuneac A, Grov C, Parsons JT. Extending the minority stress model to incorporate HIV-positive gay and bisexual men's experiences: A longitudinal examination of mental health and sexual risk behavior. Ann Behav Med. 2017;51(2):147-58.

98. Wu J. From "Long Yang" and "Dui Shi" to Tongzhi: Homosexuality in China. J Gay Lesbian Psychother. 2003;7(12):117-43

99. Cao J, Guo L. Chinese "Tongzhi” community, civil society, and online activism. Commun Public. 2017;1(4):504-8.

100. Katz-Wise SL, Rosario M, Tsappis M. LGBT youth and family acceptance. Pediatr Clin N Am. 2016;63(6):1011-25.

101. Beijing LGBT Centre. Being LGBTI in China: A national survey on social attitudes toward sexual orientation, gender identity and gender expression. United Nations Development Programme; 2016.

102. Sun S, Hoyt WT, Pachankis JE. Sexual risk behaviors in the internet age: The case of Chinese men who have sex with men. AIDS Care. 2019; (Sep): 1-8.

103. Sun S, Budge SL, Shen W, Xu G, Liu M, Feng S. Minority stress and health: A grounded theory exploration among men who have 
sex with men in China and implications for health research and interventions. Soc Sci Med. 2020; In press.

104. Lin K, Button DM, Su M, Chen S, Lin K. Chinese college students' attitudes toward homosexuality: Exploring the effects of traditional culture and modernizing factors. Sex Res Soc Policy. 2016;19:158-72.

105. Liu M. Two gay men seeking two lesbians: An analysis of Xinghun (formality marriage) ads on China's Tianya.cn. Sex Cult. 2013;17(3):494-511

106. Huang Y, Li P, Lai Z, Jia X, Xiao D, Wang T, et al. Association between sexual minority status and suicidal behavior among Chinese adolescents: A moderated mediation model. J Affect Disord. 2018;239(May):85-92.

107. Holt MK, Vivolo-Kantor AM, Polanin JR, Holland KM, DeGue S, Matjasko JL, et al. Bullying and suicidal ideation and behaviors: A meta-analysis. Pediatrics. 2015;135(2):e496-509.

108. O’Malley Olsen E, Kann L, Vivolo-Kantor A, Kinchen S, McManus T. School violence and bullying among sexual minority high school students, 2009-2011. J Adolesc Health. 2014;55(3): 432-8.

109. Hatzenbuehler ML. How does sexual minority stigma "get under the skin"? A psychological mediation framework. Psychol Bull. 2009;135(5):707-30.

110. Newcomb ME, Mustanski B. Internalized homophobia and internalizing mental health problems: A meta-analytic review. Clin Psychol Rev. 2010;30(8):1019-29.

111. Herrick AL, Stall R, Chmiel JS, Guadamuz TE, Penniman T, Shoptaw S, et al. It gets better: Resolution of internalized homophobia over time and associations with positive health outcomes among MSM. AIDS Behav. 2013;17(4):1423-30.

112. Shao J, Chang ES, Chen C. The relative importance of parentchild dynamics and minority stress on the psychological adjustment of LGBs in China. J Couns Psychol. 2018;65(5):598-604.

113. Dawson NV, Weiss R. Dichotomizing continuous variables in statistical analysis: A practice to avoid. Med Decis Mak. 2012;32(2): 225-6.

114. Altman DG, Royston P. The cost of dichotomising continuous variables. BMJ. 2006;332(7549):1080.

115. Sheridan DJ. Sexual orientation disclosure and concealment: A model-driven meta-analysis. University of Miami. Available from ProQuest Dissertations \& Theses Global.; 2017.

116. Guo Y, Li X, Liu Y, Jiang S, Tu X. Disclosure of same-sex behavior by young Chinese migrant men: Context and correlates. Psychol Health Med. 2014;19(2):190-200.

117. Sun S, Whiteley L, Brown LK. HIV testing among Chinese men who have sex with men: The roles of HIV knowledge, online social life, and sexual identity concerns. AIDS Behav. 2019. (Online First Publication).

118. Pyun T, Santos GM, Arreola S, Do T, Hebert P, Beck J, et al. Internalized homophobia and reduced HIV testing among men who have sex with men in china. Asia-Pacific J Public Heal. 2014;26(2):118-25.

119. Millar BM, Wang K, Pachankis JE. The moderating role of internalized homonegativity on the efficacy of LGB-affirmative psychotherapy: Results from a randomized controlled trial with young adult gay and bisexual men. J Consult Clin Psychol. 2016;84(7): $565-70$.

120. Chaudoir SR, Wang K, Pachankis JE. What reduces sexual minority stress? A review of the intervention "toolkit.". J Soc Issues. 2017;73(3):586-617.

121. Kitayama S, Markus HR, Kurokawa M. Culture, emotion, and well-being: Good feelings in Japan and the United States. Cognit Emot. 2000;14(1):93-124.

122. Suh EM. Culture, identity consistency, and subjective well-being. J Pers Soc Psychol. 2002;83(6):1378-91.
123. Markus HR. Kitayama S. Cultures and selves: A cycle of mutual constitution. 2010:420-30.

124. Huang S, Brouwer DC. Coming out, coming home, coming with: Models of queer sexuality in contemporary China. J Int Intercult Commun. 2017;11(2):97-116.

125. Rendina HJ, Millar BM, Parsons JT. The critical role of internalized HIV-related stigma in the daily negative affective experiences of HIV-positive gay and bisexual men. J Affect Disord. 2017;227(September 2017):289-97.

126. Wohl AR, Galvan FH, Carlos JA, Myers HF, Garland W, Witt MD, et al. A comparison of MSM stigma, HIV stigma and depression in HIV-positive latino and African American men who have sex with men (MSM). AIDS Behav. 2013;17(4):1454-64.

127. Liu C, Zhang Y, Pan SW, Wu D, Cao B, Fu R, et al. Anticipated HIV stigma among men who have sex with men and transgender individuals in China: a cross-sectional study. Lancet. 2017;390: S70.

128. Xiang Y, Yu X, Ungvari GS, Lee EHM, Chiu HFK. China's national mental health law: A 26-year work in progress. Lancet. 2012;379(9818):780-2.

129. Lin I-TS. The mental healthcare industry in China [Internet]. China Briefing. [cited 2019 Jun 17]. Available from: https:// www.china-briefing.com/news/mental-healthcare-industry-china/

130. Gao X, Jackson T, Chen H, Liu Y, Wang R, Qian M, et al. There is a long way to go: a nationwide survey of professional training for mental health practitioners in China. Health Policy. 2010;95(1): 74-81.

131. Burki T. Health and rights challenges for China's LGBT community. Lancet. 2017;389(10076):1286.

132. Beijing LGBT Center. Chinese LGBT Mental Health Survey Report. Author, editor. Beijing, China; 2014.

133. Chang DF, Tong H, Shi Q, Zeng Q. Letting a hundred flowers bloom: Counseling and psychotherapy in the People's Republic of China. J Ment Health Couns. 2005;27(2):104-16.

134. Haldeman DC. The practice and ethics of sexual orientation conversion therapy. J Consult Clin Psychol. 1994;62(2):221-7.

135. Cramer RJ, Golom FD, LoPresto CT, Kirkley SM. Weighing the evidence: Empirical assessment and ethical implications of conversion therapy. Ethics Behav. 2008;18(1):93-114.

136. Kwok DK, Winter S, Yuen M. Heterosexism in school: The counseling experience of Chinese tongzhi students in Hong Kong. Br J Guid Couns. 2012;40(5):561-76.

137. Yang JP, Simoni JM, Dorsey S, Lin Z, Sun M, Bao M, et al. Reducing distress and promoting resilience: A preliminary trial of a CBT skills intervention among recently HIV-diagnosed MSM in China. AIDS Care. 2018;30(sup5):S39-48.

138. Adam BD, Murray J, Ross S, Oliver J, Lincoln SG, Rynard V. Hivstigma.com, an innovative web-supported stigma reduction intervention for gay and bisexual men. Health Educ Res. 2011;26(5):795-807.

139. Rongkavilit C, Wang B, Naar-King S, Bunupuradah T, Parsons JT, Panthong A, et al. Motivational interviewing targeting risky sex in HIV-positive young Thai men who have sex with men. Arch Sex Behav. 2014;44(2):329-40.

140. Mimiaga MJ, Thomas B, Mayer KH, Regenauer KS, Dange A, Andres Bedoya C, et al. A randomized clinical efficacy trial of a psychosocial intervention to strengthen self-acceptance and reduce HIV risk for MSM in India: Study protocol. BMC Public Health. 2018;18(1):1-8.

141. Safren SA, Blashill AJ, O'Cleirigh CM. Promoting the sexual health of MSM in the context of comorbid mental health problems. AIDS Behav. 2011;15(SUPPL. 1):30-4.

142. Pachankis JE. A transdiagnostic minority stress treatment approach for gay and bisexual men's syndemic health conditions. Arch Sex Behav. 2015;44(7):1843-60. 
143. Diamond GM, Diamond GS, Levy S, Closs C, Ladipo T, Siqueland L. Attachment-based family therapy for suicidal lesbian, gay, and bisexual adolescents: A treatment development study and open trial with preliminary findings. Psychotherapy. 2013;49(1):62-71.

144. Leluțiu-Weinberger C, Manu M, Ionescu F, Dogaru B, Kovacs T, Dorobănțescu C, et al. An mHealth intervention to improve young gay and bisexual men's sexual, behavioral, and mental health in a structurally stigmatizing national context. J Med Internet Res. 2018;20(11):1-15.

Publisher's Note Springer Nature remains neutral with regard to jurisdictional claims in published maps and institutional affiliations. 